\title{
A Two-Layer Model for Steady-Amplitude Gravity Waves and Convection Generated by a Thermal Forcing
}

\author{
A. A. M. SAYED AND L. J. CAMPBEll \\ School of Mathematics and Statistics, Carleton University, Ottawa, Ontario, Canada
}

(Manuscript received 5 March 2017, in final form 21 February 2018)

\begin{abstract}
A two-dimensional two-layer mathematical model is described representing internal gravity waves and convection generated by a thermal forcing in the lower atmosphere. The model consists of an upper layer with stable stratification, a lower layer with unstable stratification, and a thermal forcing in the form of a nonhomogeneous term in the energy conservation equation. Exact analytical solutions are derived for some simple configurations. Depending on the vertical location and depth of the thermal forcing, the model can be used to represent different configurations in which gravity waves are generated by diabatic heating. When the thermal forcing is centered in the lower layer, convective cells are generated in the lower layer, and gravity waves are forced and propagate upward from the interface between the two layers. When the thermal forcing is centered at the interface, the convection in the lower layer is weaker, and gravity waves are forced by the direct effect of the thermal forcing in the upper layer and the influence of the convective cells below. Steadyamplitude solutions for the vertical profile of the gravity waves and convection are derived and generalized to include cases where there is a spectrum of horizontal wavenumbers or vertical wavenumbers or frequencies present.
\end{abstract}

\section{Introduction}

It has been known for decades that convection in the troposphere is an important source for internal gravity waves in the lower and middle atmosphere, but while the mechanisms for the generation of topographic gravity waves have been studied extensively (e.g., Baines 1995; Wurtele et al. 1996), the convective generation mechanisms are less well understood. Measurements and analyses quantify the relationship between convection and gravity waves in the troposphere and middle atmosphere (e.g., Pfister et al. 1986; Tsuda et al. 1990; Vincent and Alexander 2000; Alexander et al. 2008; Dutta et al. 2009) and in the upper atmosphere (e.g., Tsuda et al. 1990; Kovalam et al. 2006; Taylor et al. 2009; Venkateswara Rao et al. 2011). However, these analyses cannot give a complete description of internal gravity waves and their effects on the global circulation of the atmosphere; in particular, radar coverage is limited in location (Sato 1992), and aircraft observations provide limited information on the vertical structure of the

Corresponding author: Dr. L. J. Campbell, campbell@math. carleton.ca waves (e.g., Alexander and Pfister 1995; Alexander et al. 2000).

Additional insight into the convective generation of gravity waves has been obtained using numerical models of varying degrees of complexity. For example, Lane and Moncrieff (2008) used a two-dimensional cloudresolving model to simulate tropical convection and generate gravity waves and then carried out spectral analyses to quantify links between the characteristics of the gravity waves and the convection; Kuester et al. (2008) used numerical simulations and spectral analyses to study the case of gravity waves generated by Hurricane Humberto (2001); and Jewtoukoff et al. (2013) analyzed measurements from long-duration stratospheric balloons and carried out numerical simulations, including a case study of gravity waves generated by Tropical Storm Gelane (2010).

Investigations using large-scale general circulation models (GCMs) have also helped to advance our understanding of the convective generation of gravity waves. However, gravity waves are relatively small-scale phenomena with wavelengths smaller than a few hundred kilometers, and consequently, very high spatial resolution is needed in order for GCMs to represent them accurately. This means that it is often necessary to 
parameterize the effects of the gravity waves in GCMs by adding to the equations of the model a term to represent the drag force that would have resulted from the gravity waves if they had been correctly resolved by the model (e.g., Lindzen 1981; Hines 1997; Alexander and Dunkerton 1999). The lack of complete understanding of the mechanisms for the convective generation of gravity waves adds to the challenge of developing accurate parameterizations of the wave drag. This issue has been explored using analyses based on linear theory (e.g., Chun and Baik 2002; Chun et al. 2004; Beres 2005; Beres et al. 2005) and by numerical simulations using GCMs (e.g., Alexander et al. 1995; Pandya and Alexander 1999; Piani et al. 2000; Song et al. 2003).

It is generally understood (e.g., Pandya and Alexander 1999; Lane et al. 2001; Beres et al. 2002; Song et al. 2003; Beres et al. 2004) that convective gravity waves are generated by the combined effects of forcing from diabatic heating and nonlinear advection. Various hypotheses have been put forward for the interactions between diabatic heating, convection, and gravity waves, and three simplified descriptions have been suggested, summarized as follows by Beres et al. (2002) and Fritts and Alexander (2003):

1) A deep heating mechanism in which the gravity waves are mainly excited by a thermal forcing with no shear. In this mechanism, the dominant vertical wavelength of the excited tropospheric gravity waves is taken to be proportional to the buoyancy frequency, and it is suggested that it is approximately twice the depth of the heating (e.g., Salby and Garcia 1987; Bergman and Salby 1994; Alexander et al. 1995; Piani et al. 2000).

2) An obstacle effect mechanism, which acts in a similar manner to the mechanism for the generation of topographic gravity waves where the topography or mountain acts as an obstacle but with the convective cells playing the role of the obstacle (e.g., Clark et al. 1986; Pfister et al. 1993).

3) A mechanical oscillator mechanism in which updraft and downdraft oscillations produce vertically propagating gravity waves in a manner similar to the actions of a mechanical oscillator in a stratified fluid (e.g., Fovell et al. 1992; Lane et al. 2001).

In reality, the three mechanisms are not fully exclusive and may coexist. Clark et al. (1986), for example, found that gravity waves in a stable layer overlying convection can be generated by both the deep heating and the obstacle effect mechanisms. Moreover, nonlinear advection plays a significant role in the wave generation process (Lane and Moncrieff 2008; Song et al. 2003) and is crucial to our understanding of convective gravity waves.
Although GCM simulations and modeling have been able to examine and interpret hypotheses for the convective generation of gravity waves, the high level of complexity and the large number of degrees of freedom involved in GCM simulations make it complicated to isolate and quantify the relationships between the gravity waves and the convection. A mathematical study based on relatively simple equations that can be solved either analytically or numerically can, in principle, allow us to investigate these relationships in a more straightforward way.

There have been a number of analytical studies of convective gravity wave dynamics in the past few decades. Sang (1991), for example, considered a two-layer model consisting of steady linear equations simplified by the Boussinesq approximation and defined in a configuration with an unstable lower layer and a stable upper layer and with a thermal forcing centered at the interface between the layers. However, the focus of the study was on the case where the amplitude of the disturbance in the upper layer decays exponentially with height. These are trapped disturbances that would not reach higher levels of the atmosphere or have any significant effect on the background flow. Moreover, there are some unclear assumptions and omissions in the description of the investigation. Chun and Baik (1998) described a model based on a linear two-dimensional Boussinesq configuration with constant background velocity and constant buoyancy frequency, which was subsequently used for the development of gravity wave drag parameterization schemes (Chun and Baik 2002; Chun et al. 2004). Holton et al. (2002) made several contributions to this topic using a two-dimensional linear Boussinesq model with zero background velocity and constant buoyancy frequency and with a thermal forcing added as a nonhomogeneous term in the equation for conservation of energy. The configuration is based on that of Hayashi (1976), in which the thermal forcing has a half-sine-wave structure in the underlying heating region and is specified in the form of a Gaussian distribution horizontally and periodic in time. This study was subsequently extended to a multifrequency thermal forcing with constant mean velocity (Beres et al. 2004), and then to a three-dimensional configuration (Beres 2004), and subsequently used as the basis for a parameterization scheme (Beres 2005; Beres et al. 2005).

In the present investigation, we develop a model for gravity waves and convection in a two-dimensional region defined by Cartesian coordinates $x$ in the horizontal direction and $z$ in the vertical direction. The model consists of two layers: the lower boundary of the lower layer represents the surface of Earth, the upper layer extends up to infinity, and the two layers are connected 
by specified interface conditions. The model equations are based on the anelastic approximation in which the background density $\bar{\rho}$ varies with altitude in the absence of sound waves (Batchelor 1953; Ogura and Phillips 1962). In the lower layer, the background density increases with height so that there is unstable stratification, and in the upper layer, it decreases with height so that there is stable stratification.

A nonhomogeneous term is added to the energy conservation equation to represent a thermal forcing (deep heating). This generates a perturbation in the upper and lower layers. We consider a situation where the perturbation amplitude is small enough to allow linearization of the perturbation equations. The linear model is used to investigate the effects of the vertical and horizontal structures of thermal forcing on the perturbation. As a preliminary step, for the purpose of deriving analytical solutions, the thermal forcing is chosen to be a sinusoidal function of time and the horizontal variable with a steady amplitude depending on altitude; thus, a steady-amplitude perturbation is generated. The perturbation takes the form of closed convective cells in the unstable lower layer connected by the interface conditions to vertically sinusoidal gravity wave-like oscillations in the stable upper layer. The vertical component of the group velocity of the waves is positive, and the waves propagate upward from the interface. The anelastic approximation means that there are no sound waves present.

With this form of thermal forcing, the perturbation consists of a single mode with horizontal wavenumber specified by the forcing function, but a more realistic horizontal representation is obtained by considering the thermal forcing to be a horizontally localized function of $x$ such as a Gaussian distribution (section 4). In that case, the convective cells occur in a localized region in the lower layer, and the upper-layer oscillation is a horizontally localized wave packet consisting of vertically propagating modes corresponding to a continuous spectrum of horizontal wavenumbers. The model can also be extended to a situation where there is a wave packet that is localized in time and includes a continuous spectrum of frequencies.

The model is similar to that of Sang (1991), but some inconsistencies have been corrected, and more details have been added, and the focus here is on propagating gravity waves rather than on trapped disturbances.

By changing the vertical structure, the depth, and the vertical location of the thermal forcing, the model can be set to represent different configurations in which gravity waves are generated by diabatic heating. The deep heating mechanism, as described by Beres et al. (2002) and Fritts and Alexander (2003), can be obtained by considering a model with a single stable layer with the thermal forcing applied to generate waves. A combination of this with the obstacle effect mechanism can be achieved with a two-layer formulation with the thermal forcing centered either in the lower layer or at the interface between the two layers; in that case, convective cells are generated in the lower layer and influence the upper-layer gravity waves. The mechanical oscillator mechanism could be obtained by applying an oscillatory lower boundary condition to a single stable layer to generate upward-propagating gravity waves. This would be similar to the configuration used in previous studies of the temporal evolution of gravity waves where the waves are generated by fluid flow over a "wavy wall," as shown in Fig. 1 of Booker and Bretherton (1967).

These steady-amplitude linear solutions represent a physically unlikely situation where the unstable stratification and steady-amplitude convective perturbation are sustained indefinitely. To obtain a more realistic representation, the time-dependent and nonlinear terms must be retained in the perturbation equations, and the steady-amplitude solutions can then be considered as the initial state and used as a starting point for further time-dependent and nonlinear investigations of the two-layer system. Linear timedependent numerical solutions were obtained by Sayed (2014) for gravity waves generated by a thermal forcing in a single layer of stable stratification. An analytical solution was derived as well for the special case of the long-wave limit of a zero vertical-to-horizontal aspect ratio. In the limit of infinite time, these linear solutions approach a steady state, corresponding to the steady-amplitude solutions derived here. The linear approximation is only valid for early time as the nonlinear terms in the equation grow with time. Thus, nonlinear numerical simulations are required to give a correct representation of the late-time evolution of the gravity waves, their effects on the mean flow, and the development of higher harmonics.

In a layer with unstable stratification, the linear time-dependent convective solution grows with time. In the special case of the long-wave limit, for example, it was found by Sayed (2014) that the amplitude of the convective perturbation grows like $t^{-3 / 4} \exp \left(a t^{1 / 2}\right)$, where $a$ depends on altitude and on the extent of the unstable stratification. Linear numerical simulations also show a rapid growth in amplitude with time, which can only be mitigated by the addition of viscosity and heat conduction to the model. Thus, in the linear approximation, the unstable layer becomes invalid on a very short time scale, shorter than the time scale of evolution of gravity waves in the stable layer. Clearly, the nonlinear terms must be included in the model; this 
would allow the growing perturbation to modify and stabilize the background flow and eventually give rise to stable stratification. This can be investigated numerically. The linear solutions derived here and by Sayed (2014) give us some insight into the early time evolution of two-layer system, which can give us some guidance for subsequent nonlinear numerical simulations.

\section{A linear two-layer model for gravity waves and convection}

We consider a stratified fluid in a two-dimensional domain defined by Cartesian coordinates $x$ in the horizontal (west to east) direction and $z$ in the vertical direction. The fluid velocity components in the $x$ and $z$ directions are denoted, respectively, by $u$ and $w$; the fluid density by $\rho$; the pressure by $p$; and the potential temperature by $\theta$. At this point, we neglect viscosity and heat conduction but reintroduce them in some of our numerical investigations (Sayed 2014). In terms of dimensional quantities (denoted by the symbol $\star$ ), the equations for conservation of mass and momentum are

$$
\begin{aligned}
\frac{\partial \rho^{\star}}{\partial t^{\star}}+\frac{\partial}{\partial x^{\star}}\left(\rho^{\star} u^{\star}\right)+\frac{\partial}{\partial z^{\star}}\left(\rho^{\star} w^{\star}\right) & =0, \\
\rho^{\star}\left(\frac{\partial u^{\star}}{\partial t^{\star}}+u^{\star} \frac{\partial u^{\star}}{\partial x^{\star}}+w^{\star} \frac{\partial u^{\star}}{\partial z^{\star}}\right) & =-\frac{\partial p^{\star}}{\partial x^{\star}}, \\
\rho^{\star}\left(\frac{\partial w^{\star}}{\partial t^{\star}}+u^{\star} \frac{\partial w^{\star}}{\partial x^{\star}}+w^{\star} \frac{\partial w^{\star}}{\partial z^{\star}}\right) & =-\frac{\partial p^{\star}}{\partial z^{\star}}-g^{\star} \rho^{\star} .
\end{aligned}
$$

The constant $g^{\star}$ is the acceleration due to gravity. In terms of the potential temperature $\theta^{\star}$, conservation of energy is described by the first law of thermodynamics (e.g., Holton 2004):

$$
\frac{1}{\theta^{\star}}\left(\frac{\partial \theta^{\star}}{\partial t^{\star}}+u^{\star} \frac{\partial \theta^{\star}}{\partial x^{\star}}+w^{\star} \frac{\partial \theta^{\star}}{\partial z^{\star}}\right)=\frac{J^{\star}}{c_{p} T^{\star}},
$$

where $J^{\star}$ is the rate of heating per unit mass, $T^{\star}$ is the temperature, and $c_{p}$ is the specific heat at constant pressure.

Equations (1)-(4) are made nondimensional by introducing $L_{x}$ and $L_{z}$ as typical length scales in the $x$ and $z$ directions on the order of magnitude of the horizontal and vertical wavelengths of the gravity waves, $U$ and $W$ as typical velocities scales in the $x$ and $z$ directions, $R$ as a typical density scale, and $\Theta$ as a typical temperature scale. The continuity equation [(1)] implies that $L_{x} / U=$ $L_{z} / W$, and this ratio defines the corresponding time scale. The reference scale for the pressure is $R U^{2}$; this gives a balance of terms in (2). Nondimensional variables can be defined by

$$
\begin{gathered}
x=\frac{x^{\star}}{L_{x}}, \quad z=\frac{z^{\star}}{L_{z}}, \quad t=\frac{W t^{\star}}{L_{z}}=\frac{U t^{\star}}{L_{x}}, \quad u_{T}=\frac{u^{\star}}{U}, \\
w_{T}=\frac{w^{\star}}{W}, \quad \rho_{T}=\frac{\rho^{\star}}{R}, \quad p_{T}=\frac{p^{\star}}{R U^{2}}, \quad \theta_{T}=\frac{\theta^{\star}}{\Theta} .
\end{gathered}
$$

The anelastic approximation (Ogura and Phillips 1962) is made by replacing the total density with a steady height-dependent mean density $\bar{\rho}(z)$ so that the nondimensional continuity equation is

$$
\frac{\partial}{\partial x}\left(\bar{\rho} u_{T}\right)+\frac{\partial}{\partial z}\left(\bar{\rho} w_{T}\right)=0 .
$$

The nondimensional momentum equations are

$$
\begin{aligned}
\rho_{T}\left(\frac{\partial u_{T}}{\partial t}+u_{T} \frac{\partial u_{T}}{\partial x}+w_{T} \frac{\partial u_{T}}{\partial z}\right) & =-\frac{\partial p_{T}}{\partial x}, \\
\delta \rho_{T}\left(\frac{\partial w_{T}}{\partial t}+u_{T} \frac{\partial w_{T}}{\partial x}+w_{T} \frac{\partial w_{T}}{\partial z}\right) & =-\frac{\partial p_{T}}{\partial z}-g \rho_{T},
\end{aligned}
$$

where the nondimensional constant $g=g^{\star} L_{z} / U^{2}$ represents the gravitational acceleration and the nondimensional constant $\delta=L_{z}^{2} / L_{x}^{2}$ is the square of the vertical-to-horizontal aspect ratio. In the configuration studied here, $L_{z} \ll L_{x}$, so $\delta$ can be considered a small parameter for the purpose of asymptotic analyses. In the long-wave limit $\delta \rightarrow 0$, (8) gives the hydrostatic condition:

$$
\frac{\partial p_{T}}{\partial z}=-g \rho_{T}
$$

In the energy equation [(4)], the forcing function $J^{\star} / c_{p} T^{\star}$ has units of $\mathrm{s}^{-1}$; thus, the function $F^{\star}=\rho^{\star} J^{\star} / c_{p} T^{\star}$, which represents the effects of the forcing on the density, has a reference scale of $R$ divided by the time scale. The nondimensional energy equation can thus be written as

$$
\frac{1}{\theta_{T}}\left(\frac{\partial \theta_{T}}{\partial t}+u_{T} \frac{\partial \theta_{T}}{\partial x}+w_{T} \frac{\partial \theta_{T}}{\partial z}\right)=\frac{F_{T}}{\rho_{T}}
$$

where $F_{T}(x, z, t)$ is the nondimensional density-weighted forcing function.

Each of the total fluid quantities (with subscript $T$ ) in (7)-(10) is expressed as a sum of a background-flow quantity and a perturbation in the form

$$
\begin{aligned}
u_{T}(x, z, t) & =\bar{u}+\varepsilon u(x, z, t), \quad w_{T}(x, z, t)=\varepsilon w(x, z, t), \\
\rho_{T}(x, z, t) & =\bar{\rho}(z)+\varepsilon \rho(x, z, t), \\
p_{T}(x, z, t) & =\bar{p}(z)+\varepsilon p(x, z, t), \\
\theta_{T}(x, z, t) & =\bar{\theta}(z)+\varepsilon \theta(x, z, t), \quad F_{T}(x, z, t)=\varepsilon F(x, z, t),
\end{aligned}
$$

where the parameter $\varepsilon$ is the magnitude of the perturbation relative to the background flow and gives a measure of the extent of nonlinearity of the perturbation equations. We 
consider the situation where $\varepsilon \ll 1$, and linearization can thus be justified as a first approximation. We further consider a background flow, which has a constant horizontal velocity and satisfies the hydrostatic balance:

$$
\frac{d \bar{p}}{d z}=-g \bar{\rho} .
$$

From the ideal gas law, the potential temperature $\theta_{T}$ is proportional to the ratio $p_{T}^{1 / \gamma} / \rho_{T}$, where $\gamma$ is the ratio of the heat capacity at constant pressure to that at constant volume. Linearizing this relation shows that the meanflow quantities satisfy

$$
\frac{1}{\bar{\theta}} \frac{d \bar{\theta}}{d z}=\frac{1}{\gamma} \frac{1}{\bar{p}} \frac{d \bar{p}}{d z}-\frac{1}{\bar{\rho}} \frac{d \bar{\rho}}{d z}
$$

and in the linear approximation, the perturbation quantities satisfy

$$
\frac{\theta}{\bar{\theta}}=\frac{1}{\gamma} \frac{p}{\bar{p}}-\frac{\rho}{\bar{\rho}}
$$

We then linearize the momentum conservation equations, divide them by $\bar{\rho}$, and make use of (11)-(13) to express the gravitational term in the vertical momentum equation in terms of the potential temperature considering that the background potential temperature varies slowly with altitude (Sutherland 2010). This gives

$$
\left(\frac{\partial}{\partial t}+\bar{u} \frac{\partial}{\partial x}\right) u-\frac{d \bar{u}}{d z} w+\frac{\partial}{\partial x}\left(\frac{p}{\bar{\rho}}\right)=0
$$

and

$$
\delta\left(\frac{\partial}{\partial t}+\bar{u} \frac{\partial}{\partial x}\right) w+\frac{\partial}{\partial z}\left(\frac{p}{\bar{\rho}}\right)-g \frac{\theta}{\bar{\theta}}=0 .
$$

Linearizing the continuity equation suggests that we can define a density-weighted streamfunction $\psi(x, z, t)$ for the perturbed flow by

$$
\frac{\partial \psi}{\partial z}=-\bar{\rho} u, \quad \frac{\partial \psi}{\partial x}=\bar{\rho} w .
$$

The pressure gradient terms can then be eliminated from the linearized momentum equations by differentiating the horizontal momentum equation [(14)] with respect to $z$ and the vertical momentum equation [(15)] with respect to $x$ and subtracting one from the other. The resulting equation can then be written in terms of the perturbation streamfunction $[(16)]$ as

$$
\frac{1}{\bar{\rho}}\left[\left(\frac{\partial}{\partial t}+\bar{u} \frac{\partial}{\partial x}\right) \nabla^{2} \psi-\frac{\bar{\rho}^{\prime}}{\bar{\rho}}\left(\frac{\partial}{\partial t}+\bar{u} \frac{\partial}{\partial x}\right) \psi_{z}\right]-g \frac{\theta_{x}}{\bar{\theta}}=0,
$$

where $\nabla^{2}$ denotes the nondimensional Laplacian operator defined as $\nabla^{2} \psi=\delta \psi_{x x}+\psi_{z z}$, the subscripts $x$ and $z$ denote partial differentiation, and the primes denote differentiation with respect to $z$. The linearized energy equation is

$$
\left(\frac{\partial}{\partial t}+\bar{u} \frac{\partial}{\partial x}\right) \theta+\frac{\bar{\theta}^{\prime}}{\bar{\rho}} \psi_{x}=\frac{\bar{\theta}}{\bar{\rho}} F .
$$

Equations (17) and (18) can then be combined to give

$$
\left(\frac{\partial}{\partial t}+\bar{u} \frac{\partial}{\partial x}\right)^{2} \nabla^{2} \psi-\frac{\bar{\rho}^{\prime}}{\bar{\rho}}\left(\frac{\partial}{\partial t}+\bar{u} \frac{\partial}{\partial x}\right)^{2} \psi_{z}+\chi \psi_{x x}=g F_{x},
$$

where $\chi=(g / \bar{\theta})(d \bar{\theta} / d z)$.

The model is defined in the vertical domain $-h \leq z<\infty$, where $h$ is a positive constant. This interval is divided into an unstable lower layer given by $-h \leq z \leq 0$ and a stable upper layer given by $0<z<\infty$. A schematic diagram of the two-layer model is shown in Fig. 1. The background density is set to

$$
\bar{\rho}(z)=\rho_{0} e^{ \pm z / H},
$$

where $\rho_{0}$ is a constant that specifies the magnitude at the interface $z=0$. In the upper layer, the negative sign is set in the exponential, and $H$ is the scale height (e.g., Baines 1995). The relations (11) and (12) between the background potential temperature, pressure, and density imply that $\chi=N^{2}=\kappa g / H>0(\kappa=1-1 / \gamma)$, and $N=\sqrt{\chi}$ is the buoyancy frequency or Brunt-Väisälä frequency. In the lower layer, the positive sign is set in the exponential in (20) to give unstable stratification with $\chi=-\kappa g / H<0$. The expression (20) means that background density is continuous across the two layers; Sayed (2014) considers a situation in which there may be a discontinuity in the background density at the interface.

The forcing term is represented by

$$
F(x, z)=\hat{F}(z) e^{i k x}+\text { c.c. }
$$

where c.c. denotes the complex conjugate of the preceding term, $k$ is a constant, and the specified amplitude $\hat{F}(z)$ is a function that goes to zero near the ground and at high altitude (i.e., as $z \rightarrow \pm \infty$ ) in order to represent deep (or shallow) convective heating over a finite range of altitudes. Deep or shallow convective heating in the atmosphere can be represented in its simplest form as a function of the vertical variable that attains a maximum value at some central level and decays to zero as a function of height over a finite range of altitudes above and below that central 

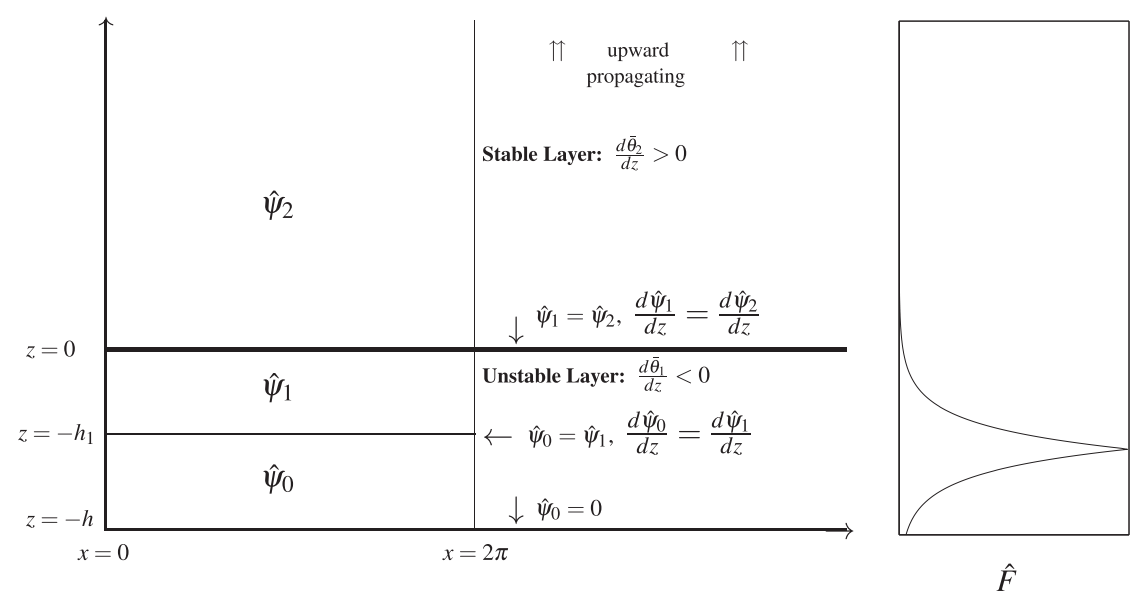

FIG. 1. A schematic diagram of the two-layer model in the domain $-h \leq z<\infty$ with the thermal disturbance centered at $z=-h_{1}$.

level. For numerical simulations, any function of this form could be specified, and the profile could be chosen based on observational measurements. In the current investigation, in order to facilitate the derivation of analytical solutions, we choose a function of the form

$$
F(x, z)=F_{0} e^{-b\left|z+h_{1}\right|} e^{i k x}+\text { c.c. }
$$

where $h_{1} \geq 0, b>0$ and $F_{0}>0,-h_{1}$ is the central level of the forcing, $b$ specifies the depth of the forcing, and $F_{0}$ specifies its strength.

The form of the forcing function $[(21)]$ means that the solution of (19) is in normal mode form:

$$
\psi(x, z, t)=\hat{\psi}(z, t) e^{i k x}+\text { c.c. }
$$

this can represent either gravity waves or convection depending on whether the stratification is stable or unstable. The amplitude $\hat{\psi}(z, t)$ satisfies

$$
\begin{aligned}
\left(\frac{\partial}{\partial t}\right. & +i k \bar{u})^{2}\left(\hat{\psi}_{z z}-\delta k^{2} \hat{\psi}\right) \mp \frac{1}{H}\left(\frac{\partial}{\partial t}+i k \bar{u}\right)^{2} \hat{\psi}_{z}-k^{2} \chi \hat{\psi} \\
& =i k g \hat{F}
\end{aligned}
$$

where the sign of the second term is negative (positive) in the lower (upper) layer. This linear time-dependent equation can be studied using asymptotic or numerical methods (Sayed 2014), but in the present paper, as a preliminary step, we consider a steady-amplitude perturbation of the form

$$
\psi(x, z)=\hat{\psi}(z) e^{i k x}+\text { c.c. }
$$

so that (24) becomes

$$
\hat{\psi}_{z z} \mp \frac{1}{H} \hat{\psi}_{z}+\left(\frac{\chi}{\bar{u}^{2}}-\delta k^{2}\right) \hat{\psi}=\frac{g}{i k \bar{u}^{2}} \hat{F} .
$$

This is a generalization of the Taylor-Goldstein equation (Taylor 1931; Goldstein 1931) that describes the amplitude of linear steady gravity waves in situations where the Boussinesq approximation is made, with the background density being approximated by a constant reference value. In the current anelastic formulation, the background stratification gives rise to the first derivative term in (26), and in addition, the equation is nonhomogeneous because of the presence of the thermal forcing.

The solution of (26) is denoted by $\hat{\psi}_{0}(z)$ for $-h \leq z<-h_{1}$ in the lower layer below the level of strongest thermal forcing, by $\hat{\psi}_{1}(z)$ for $-h_{1} \leq z \leq 0$ in the lower layer above the level of strongest thermal forcing, and by $\hat{\psi}_{2}(z)$ for $0<z<\infty$ in the upper layer.

At the bottom of the domain, a zero boundary condition,

$$
\hat{\psi}_{0}=0 \quad \text { at } \quad z=-h
$$

is imposed, and at the upper boundary, it is assumed that there is no incoming wave energy from above the domain, which means that only waves with upward group velocity are considered.

The continuity of the vertical velocity and the background density everywhere within the lower layer implies that

$$
\hat{\psi}_{0}=\hat{\psi}_{1}, \quad \frac{d \hat{\psi}_{0}}{d z}=\frac{d \hat{\psi}_{1}}{d z} \quad \text { at } \quad z=-h_{1}
$$

The continuity of the vertical velocity and the background density across the interface implies that 


$$
\hat{\psi}_{1}=\hat{\psi}_{2} \quad \text { at } \quad z=0 .
$$

Linearizing the horizontal momentum equation [(7)] tells us that the amplitude $\hat{p}$ of the pressure of a steady perturbation satisfies

$$
\hat{p}=-\frac{\bar{\rho}}{i k}\left(\frac{d \bar{u}}{d z} \hat{w}-\bar{u} \frac{d \hat{w}}{d z}\right) .
$$

With $\bar{u}$ constant and $\bar{\rho}$ continuous at the interface, the continuity of the pressure gives the interface condition

$$
\frac{d \hat{w}_{1}}{d z}=\frac{d \hat{w}_{2}}{d z} \quad \text { at } \quad z=0
$$

or, in terms of the streamfunction,

$$
\frac{d \hat{\psi}_{1}}{d z}=\frac{d \hat{\psi}_{2}}{d z} \quad \text { at } \quad z=0 .
$$

\section{Steady-amplitude expressions for the gravity waves and convection}

Equation (26) with the thermal forcing in (22) is a second-order nonhomogeneous ordinary differential equation with constant coefficients. The corresponding homogeneous equation has solutions of the form $e^{r_{1} z}$ and $e^{r_{2} z}$. In the lower layer, $-h \leq z \leq 0$, where $\chi<0, r_{1,2}$ are given by

$$
r_{1,2}=\frac{1}{2 H} \pm \sqrt{\left(\frac{1}{2 H}\right)^{2}+\lambda^{2}}
$$

where

$$
\lambda^{2}=\frac{\kappa g}{H \bar{u}^{2}}+\delta k^{2} .
$$

We examine two configurations: the first with $h_{1}>0$ so that the level $-h_{1}$ of strongest thermal forcing is below the interface (see Fig. 1) and the second with $h_{1}=0$ so that the level of strongest thermal forcing coincides with the interface (see Fig. 5). In each case, we solve for $\hat{\psi}(z)$ and then multiply it by $i k / \bar{\rho}=i k \rho_{0}^{-1} e^{-z / H}$ to obtain $\hat{w}(z)$, the amplitude of the vertical velocity perturbation.

For the case where $h_{1}>0$, we obtain

$$
\begin{aligned}
\hat{w}_{0}= & A_{0} e^{\left\{-1 /(2 H)+\sqrt{[1 /(2 H)]^{2}+\lambda^{2}}\right\} z} \\
& +B_{0} e^{\left\{-1 /(2 H)-\sqrt{[1 /(2 H)]^{2}+\lambda^{2}}\right\}} z+\frac{E e^{b h_{1}} e^{(b-1 / H) z}}{b^{2}-b / H-\lambda^{2}}
\end{aligned}
$$

for $-h \leq z<-h_{1}$ (i.e., in the lower layer below the level of strongest thermal forcing) and

$$
\begin{aligned}
\hat{w}_{1}(z)= & A_{1} e^{\left\{-1 /(2 H)+\sqrt{[1 /(2 H)]^{2}+\lambda^{2}}\right\} z} \\
& +B_{1} e^{\left\{-1 /(2 H)-\sqrt{[1 /(2 H)]^{2}+\lambda^{2}}\right\} z} \\
& +\frac{E e^{-b h_{1}} e^{-(b+1 / H) z}}{b^{2}+b / H-\lambda^{2}}
\end{aligned}
$$

for $-h_{1} \leq z \leq 0$ (i.e., in the lower layer above the level of strongest thermal forcing). The constant $E=g F_{0} /\left(\rho_{0} \bar{u}^{2}\right)$ and $A_{0}, B_{0}, A_{1}$, and $B_{1}$ can be determined from the specified boundary and interface conditions.

In the stable upper layer, $0<z<\infty, \quad \chi=N^{2}=$ $\kappa g / H>0$. We solve for $\hat{\psi}(z)$ and then multiply it by $i k / \bar{\rho}=i k \rho_{0}^{-1} e^{z / H}$ to obtain $\hat{w}(z)$. We first note that if $N^{2} / \bar{u}^{2}-\delta k^{2}=-\nu^{2}<0$, then the general solution in the upper layer is

$$
\begin{aligned}
\hat{w}_{2}= & A_{3} e^{\left\{1 /(2 H)+\sqrt{[1 /(2 H)]^{2}+\nu^{2}}\right\} z}+B_{3} e^{\left\{1 /(2 H)-\sqrt{[1 /(2 H)]^{2}+\nu^{2}}\right\} z} \\
& +\frac{E e^{-b h_{1}} e^{-(b-1 / H) z}}{b^{2}-b / H-\nu^{2}}
\end{aligned}
$$

where $A_{3}$ and $B_{3}$ are constants that can be determined from the specified boundary and interface conditions. To obtain a bounded solution as $z \rightarrow \infty$, we must have $A_{3}=0$. This situation thus gives nonoscillatory exponentially decaying trapped disturbances and is analogous to that considered by Sang (1991). If $0<N^{2} / \bar{u}^{2}-$ $\delta k^{2}<1 / 4 H^{2}$, then all the terms in the solution are nonoscillatory and grow exponentially with altitude.

The case of interest is that in which $N^{2} / \bar{u}^{2}-\delta k^{2}=$ $\mu^{2}>1 / 4 H^{2}$, which gives oscillatory gravity wave solutions. In that case, the solution includes terms proportional to $e^{i m z}$ and $e^{-i m z}$, where $m=$ $\left(N^{2} / \bar{u}^{2}-\delta k^{2}-1 / 4 H^{2}\right)^{1 / 2}$. The standard group velocity argument of Booker and Bretherton (1967, summarized in their Table 1) tells us that if $\bar{u}>0$ and $k>0$ and the square root in the definition of $m$ is taken to be positive, then the term proportional to $e^{i m z}$ corresponds to a wave with positive group velocity, while the term proportional to $e^{-i m z}$ corresponds to a wave with negative group velocity. Thus, if $\bar{u}>0$ and $k>0$, the solution of the nonhomogeneous equation that corresponds to an upward-propagating wave is

$$
\hat{w}_{2}=A_{2} e^{[1 /(2 H)] z} e^{i m z}+\frac{E e^{-b h_{1}} e^{-(b-1 / H) z}}{b^{2}-b / H+\mu^{2}}
$$

for $0<z<\infty$. The constant $A_{2}$ can be determined from the specified boundary and interface conditions. The vertical velocity $w(x, z)$ is given by $(25)$. In the complex conjugate, the horizontal wavenumber is $-k<0$, and we take the solution $e^{-i m z}$ in order to have an upwardpropagating wave. If $\bar{u}<0$, then the signs of the vertical wavenumbers are the opposite of those given here. In 
this configuration, the thermal forcing drives convection in the lower layer, which in turn generates gravity waves in the upper layer via the interface conditions.

If $h_{1}=0$, then the thermal forcing is centered at the interface $z=0$ (as shown in Fig. 5), and there is only one expression for the solution in the lower layer:

$$
\begin{aligned}
\hat{w}_{0}= & A_{0} e^{\left\{-1 /(2 H)+\sqrt{[1 /(2 H)]^{2}+\lambda^{2}}\right\} z} \\
& +B_{0} e^{\left\{-1 /(2 H)-\sqrt{[1 /(2 H)]^{2}+\lambda^{2}}\right\}}+\frac{E e^{(b-1 / H) z}}{b^{2}-b / H-\lambda^{2}}
\end{aligned}
$$

for $-h<z<0$. The constants $A_{0}$ and $B_{0}$ can be determined from the specified boundary and interface conditions. In the upper layer, the gravity wave solution takes the form (36) with $h_{1}=0$. (A schematic diagram of this configuration is shown in Fig. 5.) In this case, the thermal forcing influences both the upper and lower layers directly. In the upper layer, it generates gravity waves, and in the lower layer, it generates convection, which influences the gravity waves via the interface conditions.

The expressions (33)-(37) give the amplitude of a steady solution of the form (23) that oscillates in space only, in response to a thermal forcing of the form (21), but we can readily obtain solutions that oscillate in time as well by instead considering a thermal forcing of the form

$$
F(x, z, t)=\hat{F}(z) e^{i k(x-c t)}+\text { c.c. }=\hat{F}(z) e^{i(k x-\omega t)}+\text { c.c. }
$$

This gives a solution of the form

$$
\begin{aligned}
w(x, z, t) & =\hat{w}(z) e^{i k(x-c t)}+\text { c.c. } \\
& =\hat{w}(z) e^{i(k x-\omega t)}+\text { c.c. },
\end{aligned}
$$

where $c$ is the wave phase speed, $\omega$ is the wave frequency, and the amplitude $\hat{w}$ is given by (33)-(37) but with $\bar{u}$ replaced by $\bar{u}-c$ or $\bar{u}-\omega / k$.

These solutions can also be readily extended to a configuration where there is a spectrum of horizontal wavenumbers $k$ or phase speeds $c$ (or frequencies $\omega$ ), given by a thermal disturbance $F$ in the form of an absolutely integrable function of $x$ for which we can obtain a Fourier transform, for example, the function considered in section 4 ,

$$
F(x, z)=F_{0} e^{-b\left|z+h_{1}\right|} \frac{a^{2}}{a^{2}+x^{2}} e^{i k_{0} x}+\text { c.c. },
$$

where $a, b$, and $k_{0}$ are positive constants. The Fourier transform of $F$ in this case is

$$
\hat{F}(k, z)=\frac{a}{2} F_{0} e^{-b\left|z+h_{1}\right|} e^{-a\left|k-k_{0}\right|} .
$$

With a forcing function of this form, we seek a solution $w$ in terms of a Fourier integral:

$$
w(x, z)=\int_{-\infty}^{\infty} \hat{w}(k, z) e^{i k x} d k .
$$

For each $k$, the complex Fourier coefficient $\hat{w}(k, z)$ takes the same form as the solutions obtained above but multiplied by $(a / 2) e^{-a\left|k-k_{0}\right|}$. For each mode $k$ in the spectrum of horizontal wavenumbers, the solution in the upper layer is of the form (36), representing an oscillatory gravity wave, if

$$
|k|<k_{1}=\frac{1}{\sqrt{\delta}} \sqrt{\frac{N^{2}}{\bar{u}^{2}}-\frac{1}{4 H^{2}}}
$$

and of the form (35), representing a trapped disturbance, if $|k|>k_{1}$. Since the trapped disturbances decay exponentially with altitude, only the oscillatory modes are present at high altitudes.

In a similar manner, solutions of the form (38) can be extended to a configuration where there is a spectrum of phase speeds $c$ (or frequencies $\omega$ ), and in that form, they can be used to represent convective gravity wave drag in a parameterization scheme that could be used in a general circulation model.

\section{Graphical representation of the steady- amplitude solutions}

When the boundary and interface conditions in (27)(29) and (32) are applied and the values of the constants in the general solution of (26) are determined, the solution takes the form of convective cells in the lower layer coupled with upward-propagating gravity waves in the upper layer. In this section, the solutions are represented graphically for a situation where $H$ is large, and consequently, the terms in the exponents in the solutions arising from the first derivative term in (26) are considered small and neglected, thus simplifying the application of the boundary and interface conditions.

For the case with the thermal forcing centered at $z=-h_{1}$ in the lower layer, we obtain

$\hat{w}_{0}=\alpha \sinh \lambda(z+h)+P \frac{\cosh \lambda z}{\cosh \lambda h}+\frac{E e^{b h_{1}}}{b^{2}-\lambda^{2}} e^{(b-1 / H) z}$

for $-h \leq z<-h_{1}$,

$$
\begin{aligned}
\hat{w}_{1}(z)= & \left(\alpha \cosh \lambda h-\frac{R \cosh \lambda h_{1}}{\lambda}\right) \sinh \lambda z \\
& -i m\left(\lambda \alpha \cosh \lambda h-R \cosh \lambda h_{1}\right. \\
& +i m S-T) \cosh \lambda z+\frac{E e^{-b h_{1}}}{b^{2}-\lambda^{2}} e^{-(b+1 / H) z}
\end{aligned}
$$




$$
\begin{aligned}
& \text { for }-h_{1} \leq z \leq 0, \text { and } \\
& \begin{aligned}
\hat{w}_{2}(z)= & -\left[i m\left(\lambda \alpha \cosh \lambda h-R \cosh \lambda h_{1}+i m S-T\right)\right. \\
& -S] e^{i m z}+\frac{E e^{-b h_{1}}}{b^{2}+m^{2}} e^{-(b-1 / H) z}
\end{aligned}
\end{aligned}
$$

for $0<z<\infty$, with the constants $E, P, R, S, T$, and $\alpha$ given in the appendix. For the case with the thermal forcing centered at the interface $z=0$, we obtain

$$
\begin{aligned}
\hat{w}_{0}= & \alpha_{0} \sinh \lambda(z+h)+P_{0} \frac{\cosh \lambda z}{\cosh \lambda h} \\
& +\frac{E}{b^{2}-\lambda^{2}} e^{(b-1 / H) z}
\end{aligned}
$$

for $-h \leq z<0$ and

$$
\begin{aligned}
\hat{w}_{2}(z)= & \left(\alpha_{0} \sinh \lambda h+P_{0} \operatorname{sech} \lambda h-S_{0}\right) e^{i m z} \\
& +\frac{E}{b^{2}+m^{2}} e^{-(b-1 / H) z}
\end{aligned}
$$

for $0<z<\infty$, with the constants $E, P_{0}, S_{0}$, and $\alpha_{0}$ given in the appendix. With this simplification, $m=\sqrt{N^{2} / \bar{u}^{2}-\delta k^{2}}$ in (44), (45), and (47) and in the expressions for the constants.

We note that in evaluating the constants in (43)-(45), $w_{0}$ and $w_{1}$ are matched according to the conditions at $z=-h_{1}$ within the lower layer, and $w_{1}$ and $w_{2}$ are matched according to the conditions at the interface $z=0$; however, in evaluating the constants in (46) and (47), $w_{0}$ and $w_{2}$ are matched according to the conditions at $z=0$, and the constants in each case are thus different. This means that setting $h_{1}=0$ in the solutions (43)-(45) does not give the exact expressions in (46) and (47).

The graphs of these solutions are shown here in a rectangular domain $0 \leq x \leq 2 \pi$ and $-10 \leq z \leq 20$ with the interface at $z=0$ and the level of strongest thermal forcing at $z=-h_{1}$ with $h_{1}$ set to either 5 or 0 . The following nondimensional parameter values are set: the horizontal wavenumber $k=1$, the background velocity $\bar{u}=1$, the scale height $H=10$, the gravitational acceleration $g=3.43$, the constant $\kappa=2 / 7 \approx 0.286$, and the square of the buoyancy frequency $N^{2}=\kappa g / H=0.098$. To illustrate the main important features of the solutions, we keep $N, \bar{u}$, and $k$ fixed and plot the solutions for two different values of the aspect ratio. Setting $\delta=0.01$ gives $N^{2} / \bar{u}^{2}-\delta k^{2}>0$, which is a case with oscillatory gravity waves in the upper layer. For contrast, setting $\delta=0.1$ gives $N^{2} / \bar{u}^{2}-\delta k^{2}<0$, which is a case with trapped disturbances in the upper layer (Sang 1991).

Two configurations are shown: the first where the thermal forcing is centered at $z=-h_{1}=-5$ within the lower layer (Fig. 1) and the amplitude is given by
(43)-(45) and the second where the thermal forcing is centered at the interface $z=0$ (Fig. 5) and the amplitude is given by (46) and (47). In each case, two different thermal forcing profiles are considered: one where $b=0.7$ in (22), corresponding to shallow heating, and the other with $b=0.07$, corresponding to deep heating.

The nondimensional parameters are related to the corresponding dimensional values by (5). The scale height $H^{\star}$ in the troposphere is approximately $7 \mathrm{~km}$ (Gill 1982), so for the graphs shown here, the vertical length scale is $L_{z}=H^{\star} / H=0.7 \mathrm{~km}$ and the horizontal length scale is $L_{x}=L_{z} / \sqrt{\delta}=7 \mathrm{~km}$. The acceleration due to gravity $g^{\star}$ is approximately $9.8 \mathrm{~m} \mathrm{~s}^{-2}$, so our choices of nondimensional parameter $g=L_{z} g^{\star} / U^{2}=3.43$ and mean flow speed $\bar{u}=1$ correspond to a reference horizontal velocity of $U=\sqrt{2} \mathrm{~m} \mathrm{~s}^{-1}$ and a dimensional value of $\bar{u}^{\star}=\sqrt{2} \mathrm{~m} \mathrm{~s}^{-1}$.

Figures $2 \mathrm{a}$ and $2 \mathrm{~b}$ show the vertical velocity perturbation $w(x, z)$ as a function of altitude $z$ at fixed $x=0$ and as a contour plot for the case where the thermal forcing takes the form shown in Fig. 2c, namely, shallow heating $(b=0.7)$ centered at the altitude $z=-5$ within the lower layer. In this case, convection, with amplitude given by (43) and (44), is generated in the lower layer, and it in turn generates a disturbance in the upper layer via the interface conditions; this is an illustration of an obstacle effect occurring as a result of the thermal forcing. In this figure, $\delta=0.01$, so $N^{2} / \bar{u}^{2}-\delta k^{2}>0$ and the disturbance takes the form of upward-propagating gravity waves with amplitude given by (45). We note that the second term in (45) includes an exponential factor of $e^{-(b-1 / H) z}=e^{-0.6 z}$, which decreases with height and is thus negligible in the upper layer. Thus, for large $z$, the vertical velocity is proportional to $\cos (k x+m z)$, where $m=\sqrt{N^{2} / \bar{u}^{2}-\delta k^{2}}$. Figure 3 shows the same configuration as Fig. 2 but with $\delta=0.1$. In this case, $N^{2} / \bar{u}^{2}-\delta k^{2}<0$, so exponentially decaying trapped disturbances are generated in the upper layer, according to the expression (35).

Figures $4 \mathrm{a}$ and $4 \mathrm{~b}$ show the vertical velocity perturbation $w(x, z)$ as a function of altitude $z$ at fixed $x=0$ and as a contour plot for the case where the thermal forcing takes the form shown in Fig. 4c, namely, deep heating $(b=0.07)$ centered at the altitude $z=-5$. In this case, the thermal forcing spans a range of altitudes in both the lower and upper layers and thus directly generates gravity waves as well as convection. As before, an obstacle effect occurs as a result of the thermal forcing. Since $b-1 / H<0$, the second term in (45) is not small in this case, and the real function $w$ in the upper layer is the sum of a term proportional to $\cos (k x+m z)$ representing the gravity wave and a term proportional to $\cos (k x)$, which arises from the thermal forcing and varies exponentially with altitude. 
(a)

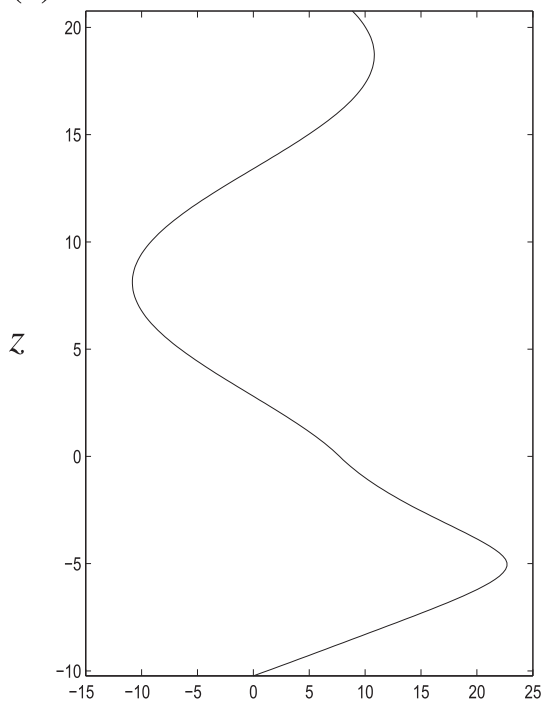

$\hat{w}$ (b)

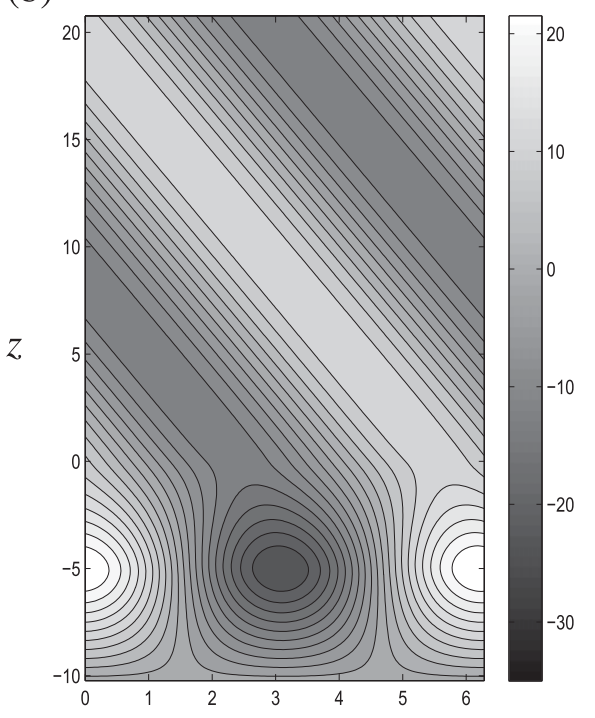

$x$ (c)

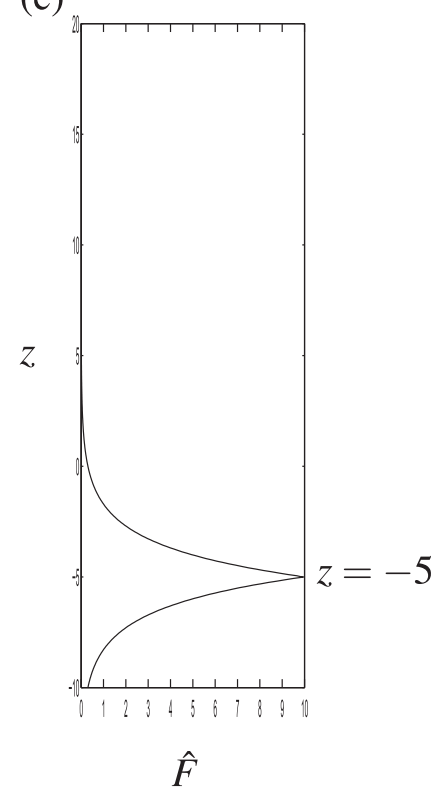

FIG. 2. (a) Vertical profile and (b) contour plot of the vertical velocity perturbation $w(x, z)$ in the two-layer model (Fig. 1) with shallow heating given by (c) a horizontally monochromatic thermal forcing function centered at $z=-5$ with vertical decay rate $b=0.7, \delta=0.01$, and $N^{2} / \bar{u}^{2}-\delta k^{2}>0$. Upward-propagating gravity waves are generated above the interface $z=0$.

The nondimensional vertical wavenumber in the configurations shown in Figs. 2 and 4 is $m=\sqrt{N^{2} / \bar{u}^{2}-\delta k^{2}} \approx 0.29$, and the nondimensional vertical wavelength is $\lambda=2 \pi / m \approx 21.2$. This corresponds to a dimensional vertical wavelength of $\lambda^{\star}=L_{z} \lambda \approx 14.7 \mathrm{~km}$. With the choice of $b=0.7$ in Fig. 2, the nondimensional heating depth $D \approx 10$, so the dimensional heating depth $D^{\star}=L_{z} D \approx 7 \mathrm{~km}$. Simulations and observational measurements (e.g., Salby and Garcia 1987; Pandya and Alexander 1999; McLandress et al. 2000) indicate that (a)

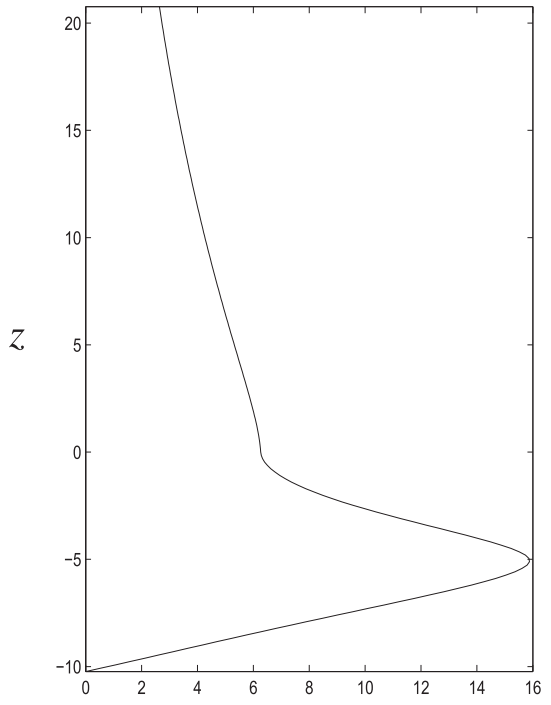

$\hat{w}$ (b)

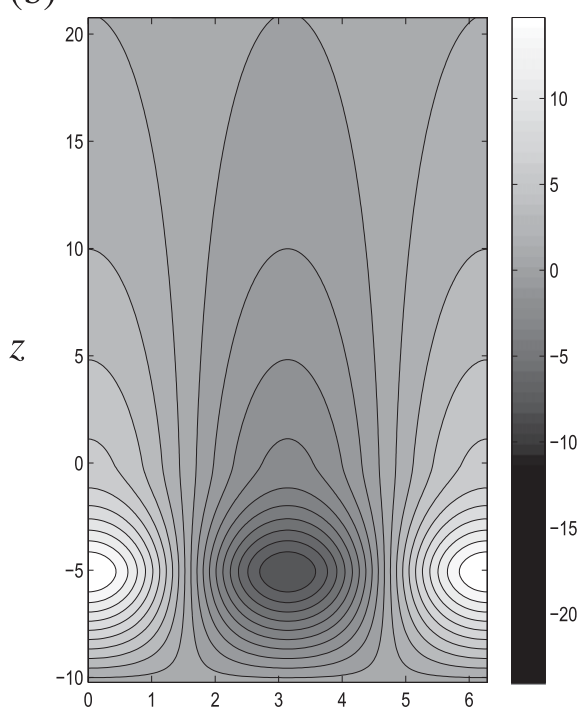

$x$ (c)

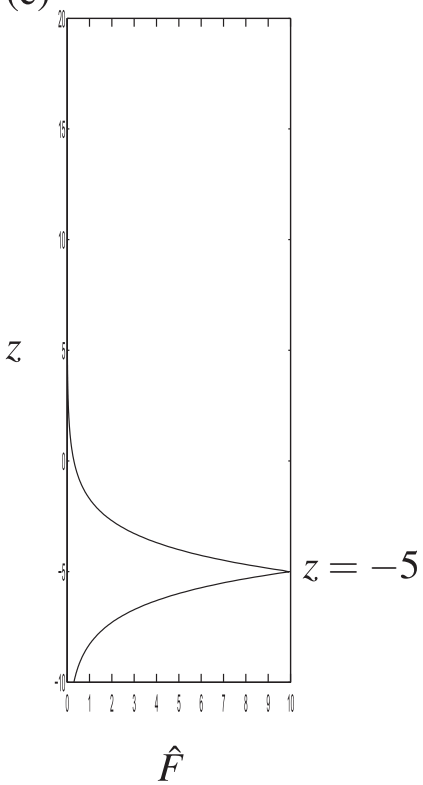

FIG. 3. As in Fig. 2, but with $\delta=0.1, N^{2} / \bar{u}^{2}-\delta k^{2}<0$. An exponentially decaying trapped disturbance is generated above the interface $z=0$. 
(a)

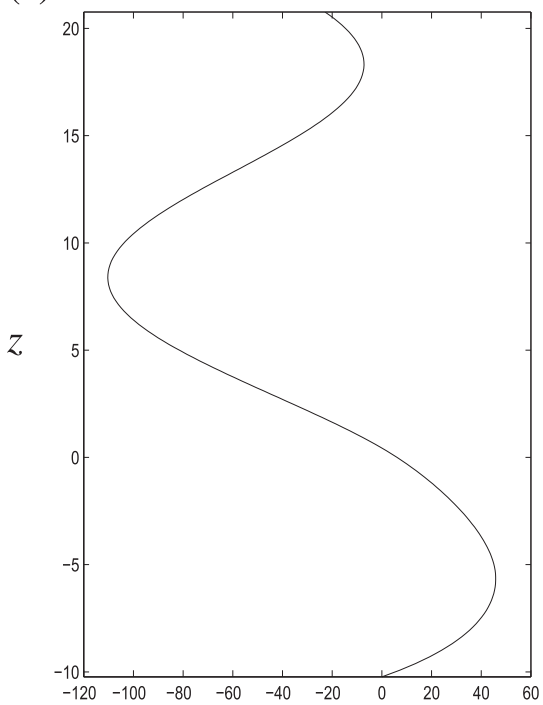

$\hat{w}$ (b)

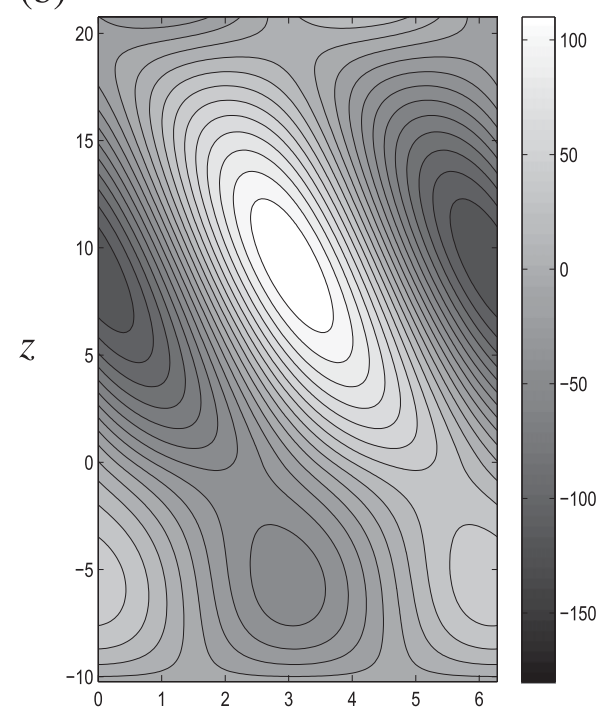

$x$ (c)

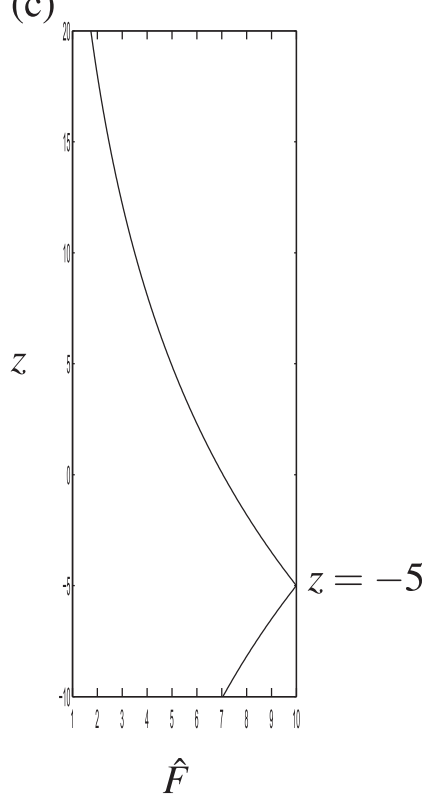

FIG. 4. (a) Vertical profile and (b) contour plot of the vertical velocity perturbation $w(x, z)$ in the two-layer model (Fig. 1) with deep heating given by (c) a horizontally monochromatic thermal forcing function centered at $z=-5$ with vertical decay rate $b=0.07, \delta=0.01$, and $N^{2} / \bar{u}^{2}-\delta k^{2}>0$. Upward-propagating gravity waves are generated above the interface $z=0$.

the vertical wavelength of convectively generated gravity waves is generally about twice the depth of the heating. Thus, the choice of input nondimensional parameters used to illustrate our solution is realistic compared with the real atmosphere. It is important to note, however, that the gravity wave vertical wavelength does not depend on the depth of the heating. Clearly, $m$ depends on $N^{2}=\kappa g / H$ and on $\bar{u}, \delta$, and $k$ but not on $b$. Changing the depth of the heating (by changing $b$ ) does not affect the vertical wavelength $2 \pi / \mathrm{m}$. This is consistent with the suggestion that the gravity wave vertical wavelength does not depend on the vertical structure of the diabatic forcing noted by Holton et al. (2002) and Lane and Moncrieff (2008).

Changing the depth of the heating does, however, change the overall structure of the upper-layer perturbation. If the heating is shallow (large $b$ ), then over the range of low altitudes corresponding to the heating depth, the solution is a linear combination of $\cos (k x+m z)$ and $\cos (k x)$, and at higher altitudes, it takes the form $\cos (k x+m z)$. If the heating is deep, then the solution is a linear combination of $\cos (k x+m z)$ and $\cos (k x)$ over a wider range of altitudes corresponding to the heating depth. In particular, if $b=1 / H$, then the solution is a linear combination of $\cos (k x+m z)$ and $\cos (k x)$ at all levels.

Figure 5 is similar to Fig 1, except with the thermal forcing centered at the interface $z=0$. Figures 6 and 7 show the vertical velocity perturbation $w(x, z)$ as a function of altitude $z$ at fixed $x=0$ and as a contour plot for the case where the thermal forcing is centered at the interface $z=0$ with shallow heating $(b=0.7)$ and with deep heating $(b=0.07)$ and with the same input parameter values as in the previous figures. The solution takes the form (47) in the upper layer, representing gravity waves and thermal effects, and it takes the form (46) in the lower layer, representing convection, but weaker than what was shown in Figs. 2 and 4 when the thermal forcing was centered below the interface within the lower layer. As before, changing the depth of the thermal forcing by changing $b$ changes the structure of the upper-layer perturbation. For shallow heating, the solution takes the form $\cos (k x+m z)$, and for deep heating, it is a linear combination of $\cos (k x+m z)$ and $\cos (k x)$.

Figures 8-12 show solutions obtained for gravity wave packets and convection generated by a thermal "island," localized in the horizontal as well as vertical direction. The forcing term takes the form (39) with $h_{1}=5$ or $h_{1}=0$ and with $b=0.7$ (shallow heating) or $b=0.07$ (deep heating). With $F$ given by (39) and its Fourier transform $\hat{F}$ given by (40), the vertical velocity perturbation takes the form (41). For each $k$, the complex Fourier coefficient $\hat{w}(k, z)$ takes the same form as (43)-(45), (46), and (47) but multiplied by a factor of $(a / 2) e^{-a\left|k-k_{0}\right|}$ in each case. In the upper layer, the Fourier coefficient is either of the form (45) or (47) representing an oscillatory gravity wave mode if $N^{2} / \bar{u}^{2}-\delta k^{2}>0$ and of the form (35) representing a trapped disturbance 

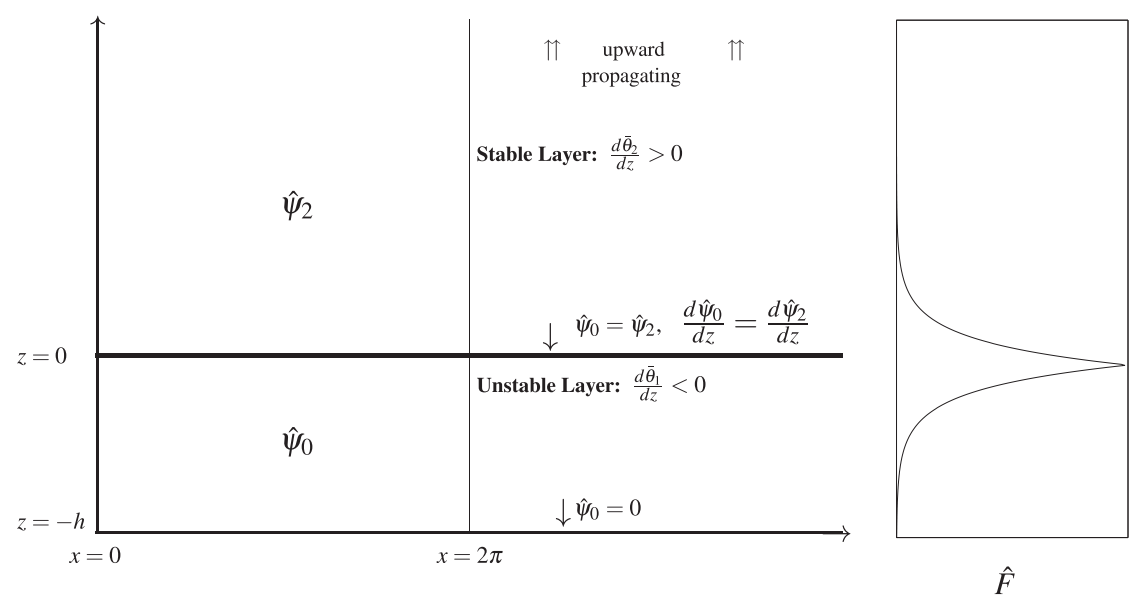

FIG. 5. As in Fig. 1, but for the thermal disturbance centered at the interface $z=0$.

mode if $N^{2} / \bar{u}^{2}-\delta k^{2}<0$. Thus, $k_{1}=N / \sqrt{\delta} \bar{u}$ is the high wavenumber cutoff point for oscillatory gravity waves. At high altitudes, only the oscillatory modes are nonnegligible. The constant $a$ in (39) determines the horizontal extent of the wave packet envelope; in the figures shown, it is set to $a=0.7$. The central wavenumber $k_{0}$ of wave packet spectrum determines the horizontal wavelength and number of oscillations within the wave packet. With $k_{0}=0$, there is a single convective cell in the lower layer and a gravity wave in the upper layer that resembles a topographic wave generated by an isolated mountain. If $k_{0}$ is nonzero, then there can be multiple convective cells in the lower layer and a gravity wave packet in the upper layer with one or more horizontal wavelengths within the horizontal extent of packet. These horizontally localized solutions are shown in the rectangular domain $-2 \pi \leq x \leq 2 \pi$ and $-10 \leq z \leq 20$, with the background velocity set to $\bar{u}=\sqrt{2}$, the square of the aspect ratio set to $\delta=0.001$, and the central wavenumber of the forcing function [(39)] set to $k_{0}=1$. The other input parameters are the same as those used in Figs. 2-7. (a)

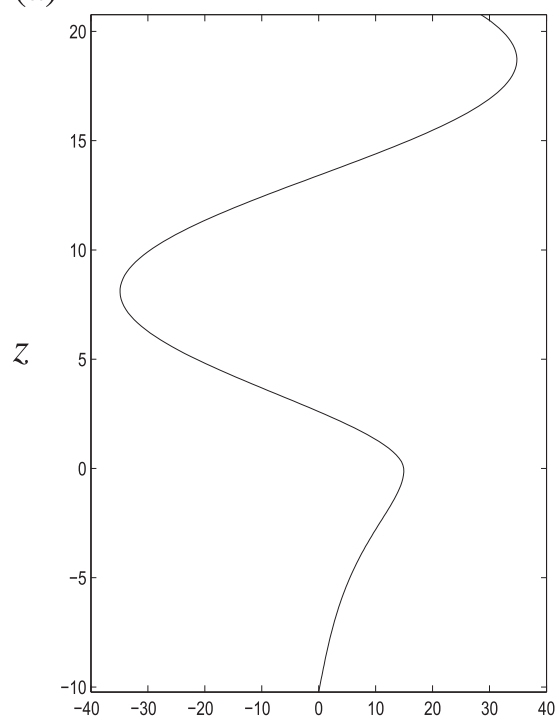

$\hat{w}$ (b)

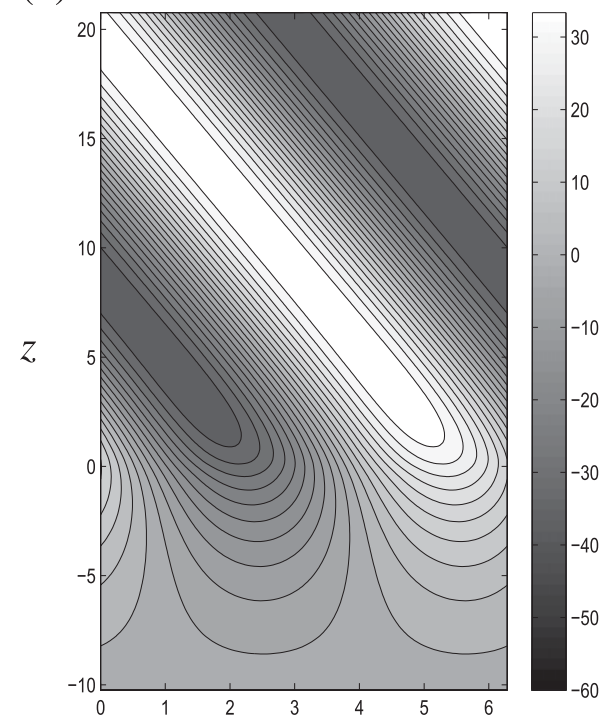

$x$ (c)

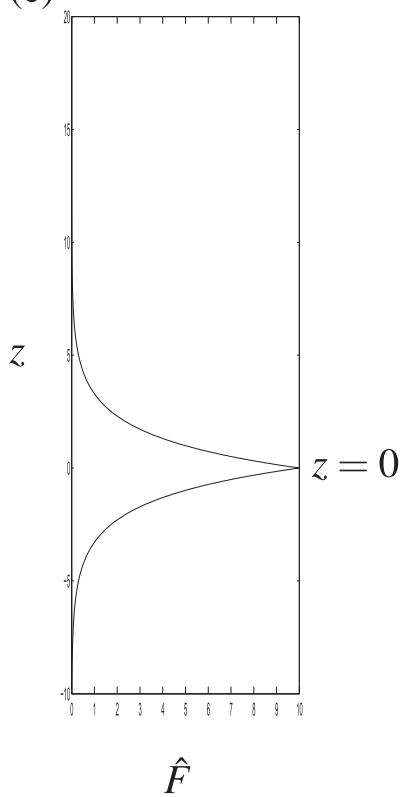

FIG. 6. (a) Vertical profile and (b) contour plot of the vertical velocity perturbation $w(x, z)$ in the two-layer model (Fig. 5) with shallow heating given by (c) a horizontally monochromatic thermal forcing function centered at $z=0$ with vertical decay rate $b=0.7, \delta=0.01$, and $N^{2} / \bar{u}^{2}-\delta k^{2}>0$. Upward-propagating gravity waves are generated above the interface $z=0$. 
(a)

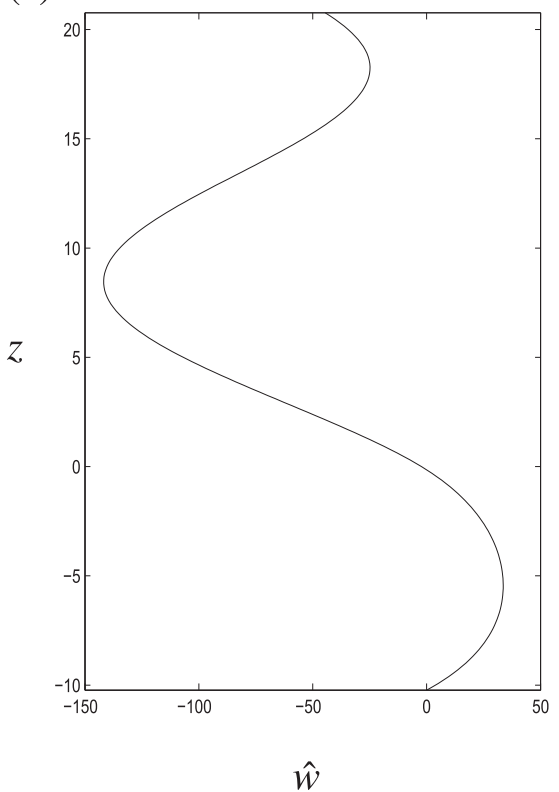

(b)

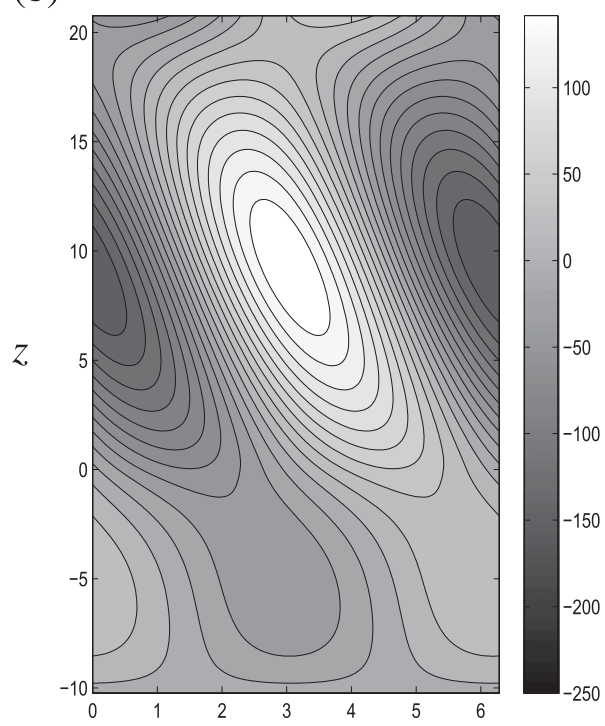

$x$ (c)

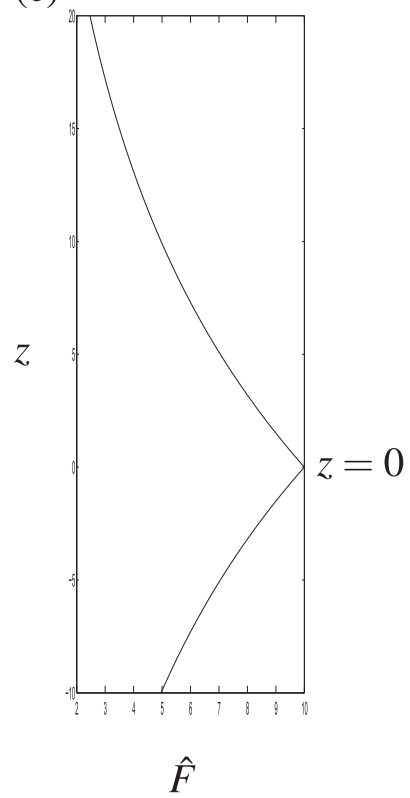

FIG. 7. (a) Vertical profile and (b) contour plot of the vertical velocity perturbation $w(x, z)$ in the two-layer model (Fig. 5) with deep heating given by (c) a horizontally monochromatic thermal forcing function centered at $z=0$ with vertical decay rate $b=0.07, \delta=0.01$, and $N^{2} / \bar{u}^{2}-\delta k^{2}>0$. Upward-propagating gravity waves are generated above the interface $z=0$.

In Fig. 8, the thermal forcing is centered at $z=-h_{1}=-5$ and $b=0.7$, so the heating is shallow. With $k_{0}=1$, the horizontal wavelength is $2 \pi$; thus, one-half of a wavelength is approximately the same as the width of the packet, so there is only one convective cell. A horizontally localized gravity wave packet is generated above the convective cell by an obstacle effect resulting from the thermal forcing. Figure 9 shows the amplitude of the (a)

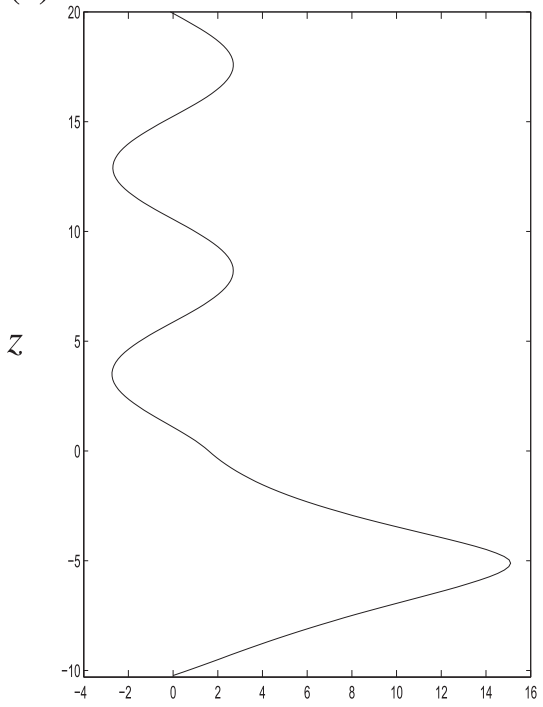

$\hat{w}$ (b)

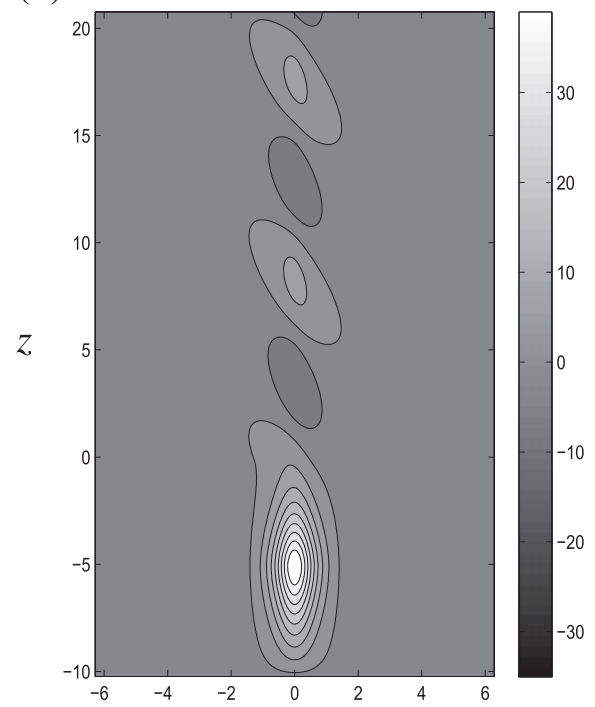

$x$ (c)

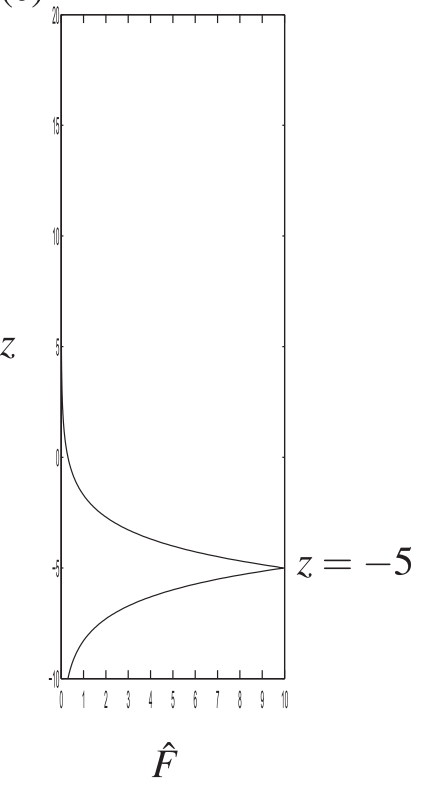

FIG. 8. (a) Vertical profile and (b) contour plot of the vertical velocity perturbation $w(x, z)$ in the two-layer model (Fig. 1) with deep heating given by (c) a horizontally localized thermal forcing function centered at $z=-5$ with vertical decay rate $b=0.7$. A horizontally localized gravity wave packet is generated above the interface $z=0$. 
(a)
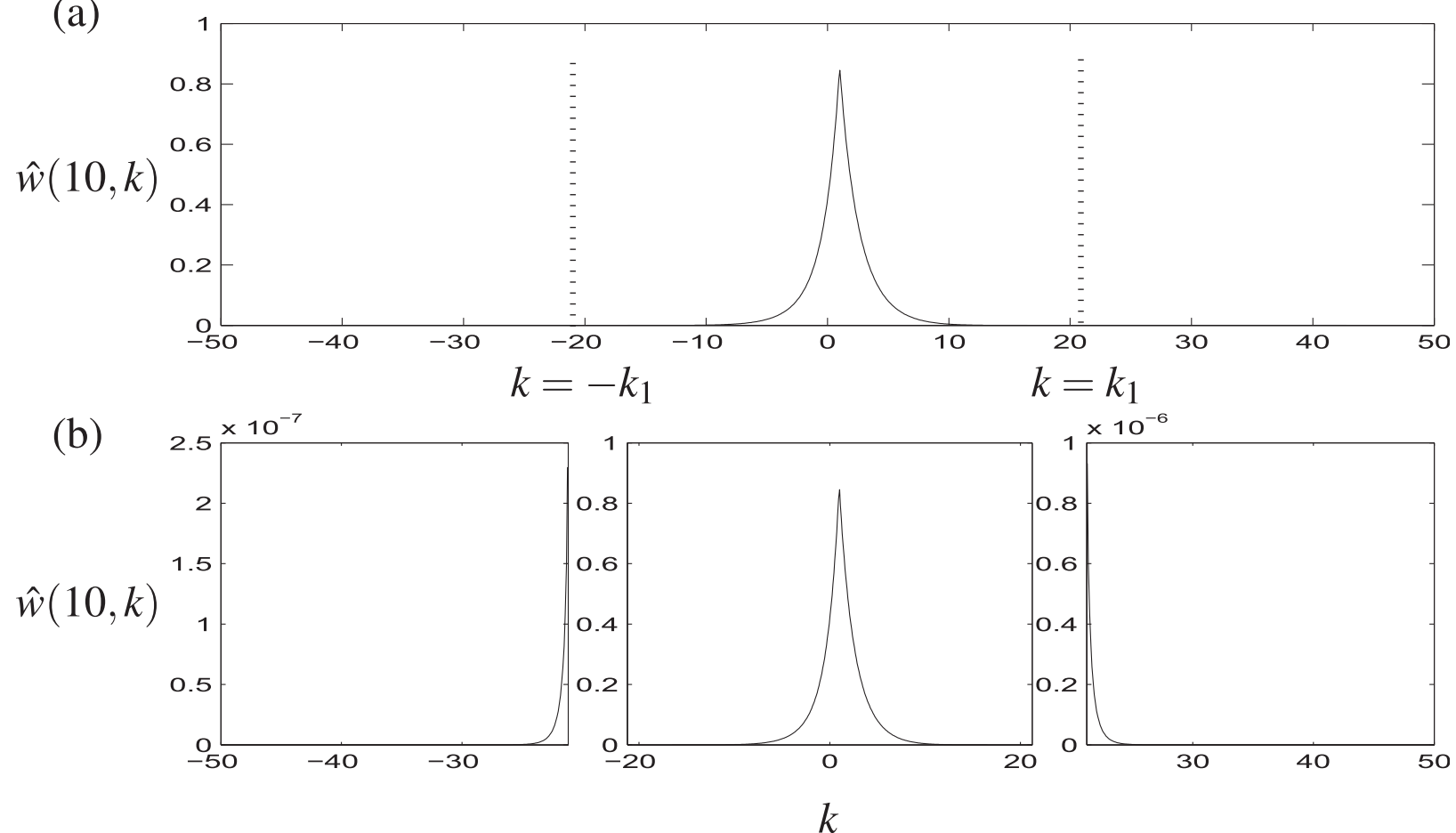

FIG. 9. (a) The amplitude of the vertical velocity perturbation $\hat{w}(z, k)$ as a function of horizontal wavenumber $k$ at $z=10$ for the case with a horizontally localized thermal forcing. The cutoff wavenumber $k= \pm k_{1}$ is indicated by the vertical bars. (b) The high-wavenumber modes $\left(|k|>k_{1}\right)$ shown on a different scale.

Fourier transform of the vertical velocity perturbation $\hat{w}(k, z)$ as a function of the horizontal wavenumber $k$ at $z=10$ in the upper layer. The cutoff wavenumber for oscillatory waves $k_{1}=N / \sqrt{\delta} \bar{u} \approx 22$. The dominant contribution to the solution comes from the wavenumbers $|k|<k_{1}$. The modes corresponding to $|k|>k_{1}$ decay exponentially with altitude; at $z=10$, the amplitude of these modes is of order $10^{-7}$. In Fig. 10, the thermal forcing is centered at $z=-h_{1}=-5$ and $b=0.07$, so there is deep heating, which generates a gravity wave packet over a single convective cell.

In Figs. 11 and 12, the thermal forcing is centered at $z=0$ with $b=0.7$ (shallow heating) in Fig. 11 and $b=0.07$ (deep heating) in Fig. 12. With the thermal forcing centered at $z=0$, the convection is weaker and the gravity wave amplitude is larger than in the corresponding configuration with the thermal forcing centered below the interface. By examining the spectrum of the solution in each case, we note again that the dominant contributions to the solution are the oscillatory gravity wave modes with horizontal wavenumbers $|k|<k_{1}$. The solutions obtained here can be extended in a similar manner to represent the situation where there is a continuous spectrum of phase speeds representing a wave packet that is localized in time as well as in space.

\section{Conclusions}

A simple two-layer model for gravity waves and convection was presented here, and steady-amplitude solutions were derived and illustrated in graphical form for several different configurations. The model includes a thermal forcing representing shallow or deep heating with the forcing centered either at some level within the lower layer or at the interface between the two layers. The thermal forcing function can be either horizontally periodic or horizontally localized. In the case where the thermal forcing is centered within the lower layer, strong convection is generated in the lower layer and generates gravity waves in the upper layer. In the case where the thermal forcing is centered at the interface, the convection is weaker, the thermal forcing influences both layers directly, and gravity waves are generated in the upper layer by the thermal forcing and by the convection from below.

The depth of the thermal forcing is defined by the parameter $b$ in (22), and it determines the structure of the gravity waves in the upper layer. When the thermal forcing is shallow (relatively large $b$ ), the gravity waves take the approximate form $\cos (k x+m z)$. When the thermal forcing is deep (relatively small $b$ ), there is a linear superposition of the gravity wave form 
(a)

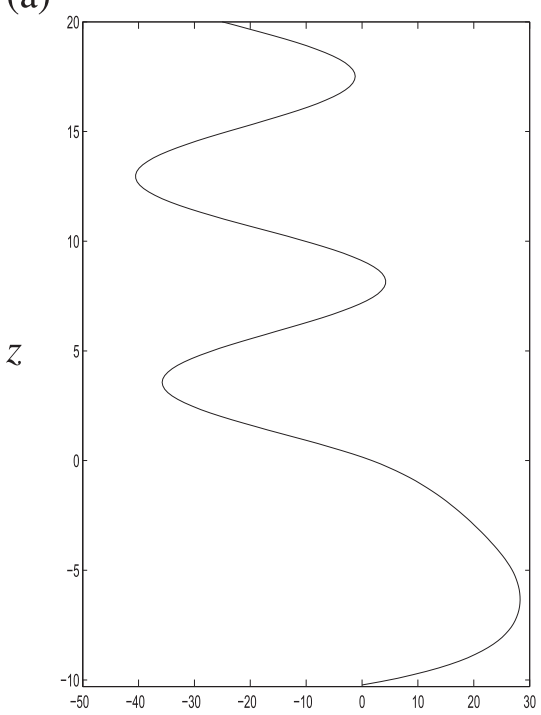

$\hat{w}$ (b)

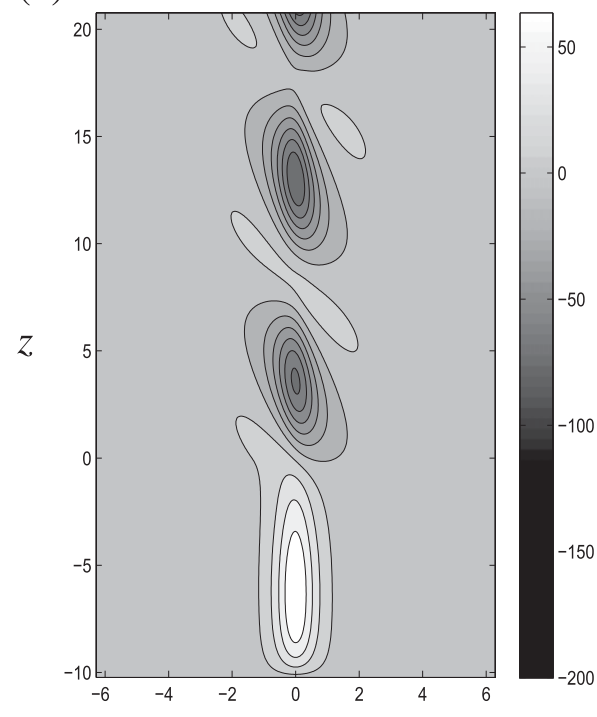

$x$ (c)

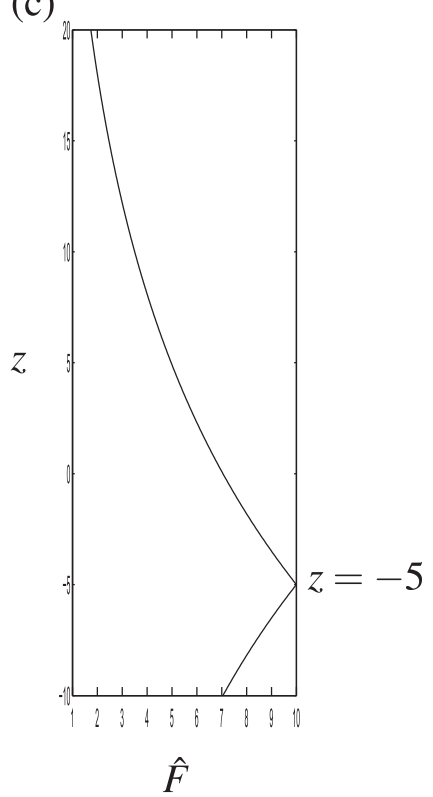

FIG. 10. (a) Vertical profile and (b) contour plot of the vertical velocity perturbation $w(x, z)$ in the two-layer model (Fig. 1) with deep heating given by (c) a horizontally localized thermal forcing function with vertical profile centered at $z=-5$ with vertical decay rate $b=0.07$. A horizontally localized gravity wave packet is generated above the interface $z=0$.

$\cos (k x+m z)$ and a function proportional to $\cos (k x)$, which arises from the thermal forcing.

Previous studies such as Sang (1991), Chun and Baik (1998), Holton et al. (2002), Beres (2004), and Beres et al. (2004) examined configurations similar to ours but made the Boussinesq approximation where the density is set to a constant $\rho_{0}$ everywhere except in the buoyancy term and the buoyancy frequency $N$ is set to a constant. (a)

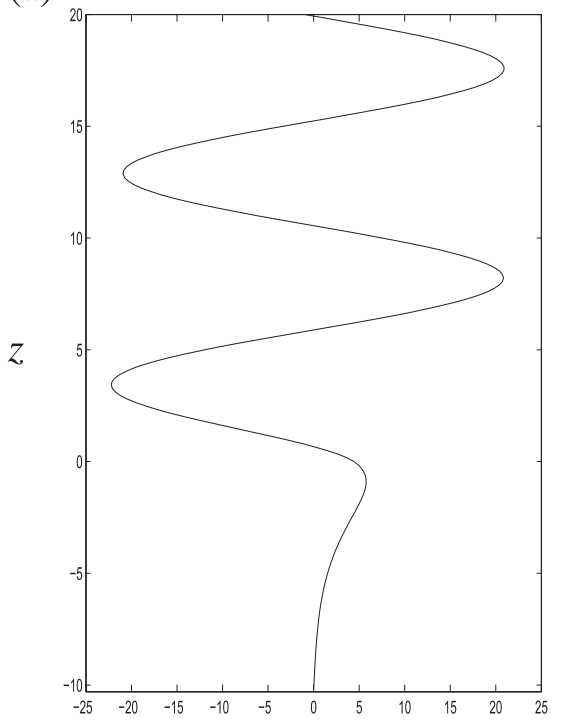

$\hat{w}$ (b)

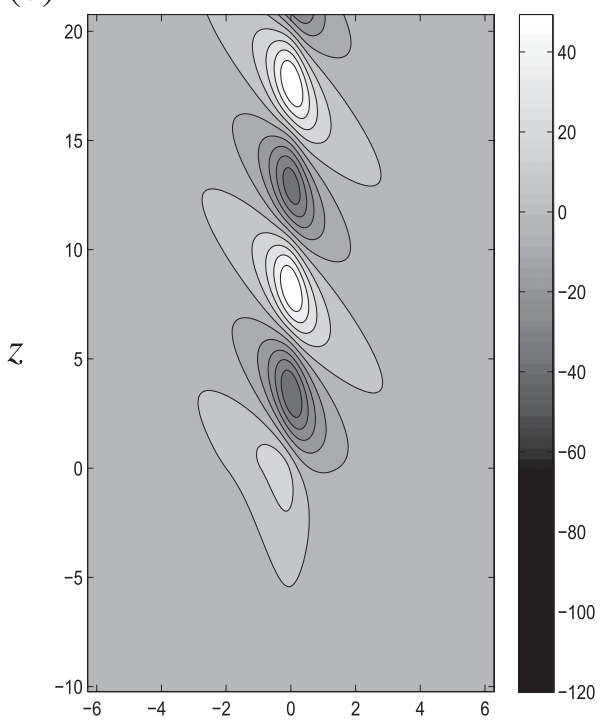

$x$ (c)

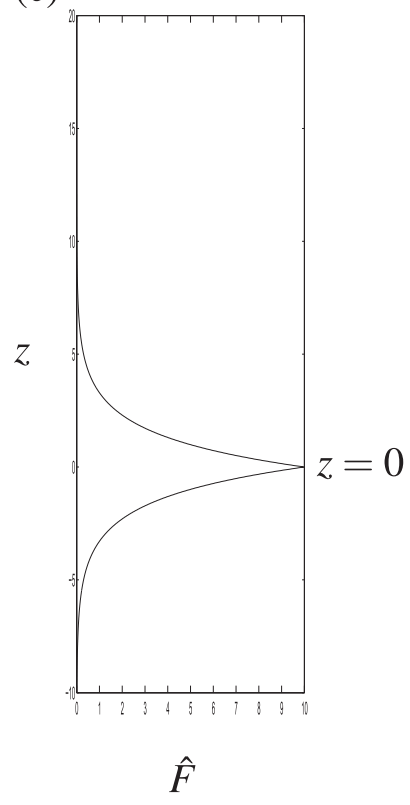

FIG. 11. (a) Vertical profile and (b) contour plot of the vertical velocity perturbation $w(x, z)$ in the two-layer model (Fig. 5) with deep heating given by (c) a horizontally localized thermal forcing function centered at $z=0$ with vertical decay rate $b=0.7$. A horizontally localized gravity wave packet is generated above the interface $z=0$. 
(a)

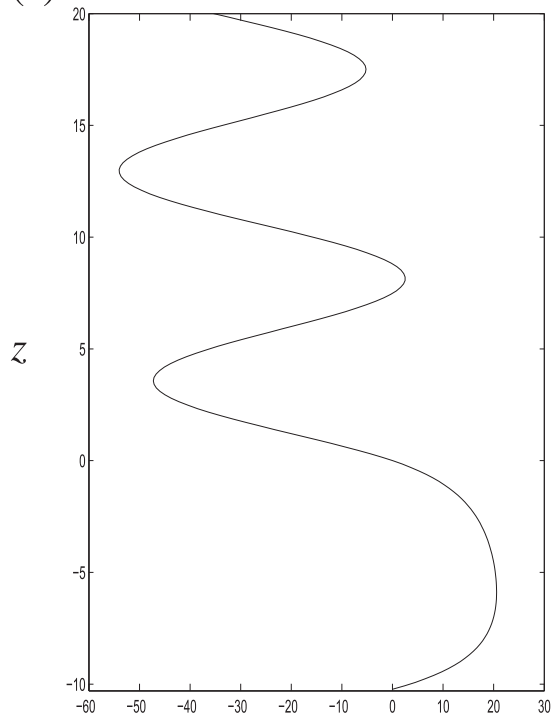

$\hat{w}$ (b)

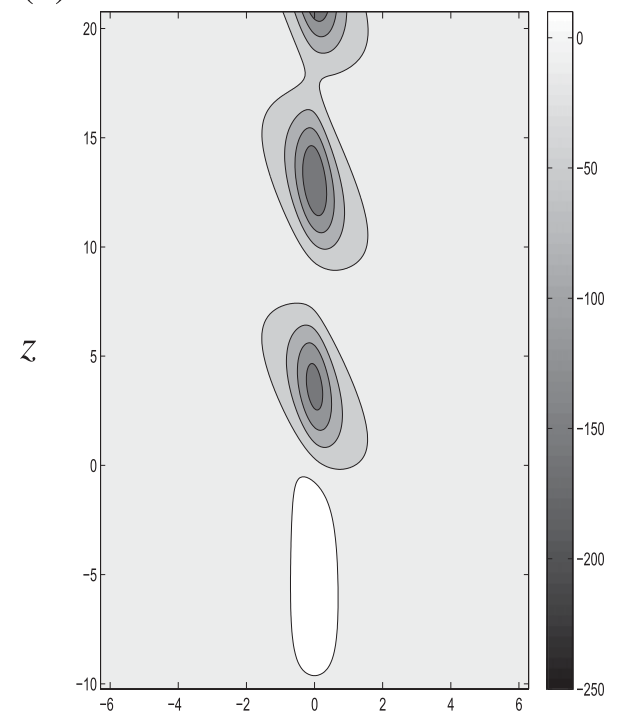

$x$ (c)

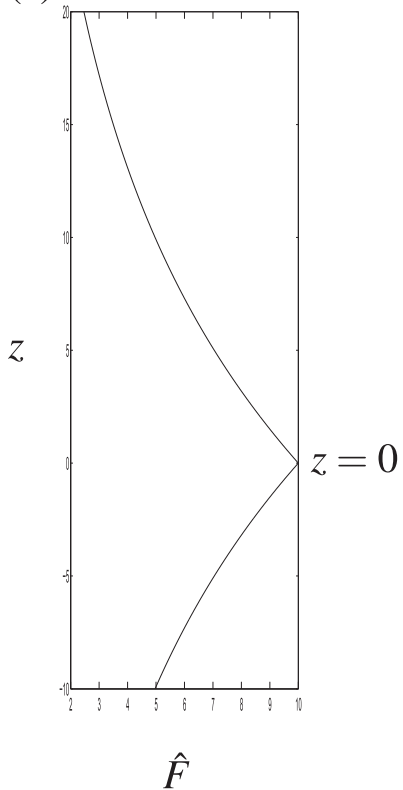

FIG. 12. (a) Vertical profile and (b) contour plot of the vertical velocity perturbation $w(x, z)$ in the two-layer model (Fig. 5) with deep heating given by (c) a horizontally localized thermal forcing function centered at $z=0$ with vertical decay rate $b=0.07$. A horizontally localized gravity wave packet is generated above the interface $z=0$.

In these studies, $N^{2}=-\left(g / \rho_{0}\right)(d \bar{\rho} / d z)$ in the whole domain, so there was stable stratification everywhere and no convection. In contrast, we made the anelastic approximation with the background density profile in the form of an exponential function of $z$ and with stable stratification in the upper layer and unstable stratification in the lower layer, thus allowing the existence of convective cells in the lower layer.

We also considered the situation where gravity wave packets and convection are generated by a thermal forcing that is localized in the horizontal as well as vertical direction, for example, where the thermal forcing is a Gaussian function of the horizontal variable. In that case, the solution includes a continuous spectrum of horizontal wavenumbers $k$, and the dominant contribution to the wave packet consists of oscillatory modes corresponding to horizontal wavenumbers $|k|<k_{1}$, given by (42). The modes corresponding to horizontal wavenumbers $|k|>k_{1}$ take the form of trapped disturbances that decay exponentially with altitude.

These gravity wave analytical solutions could be used as the basis for a gravity wave drag parameterization scheme for use in general circulation models. In parameterization schemes (e.g., Beres 2005; Beres et al. 2005), the gravity waves are generally assumed to take the form of a spectrum of vertical wavenumbers or phase speeds representing a wave packet localized in time. A representation of this form is readily made by assuming that the disturbance is a periodic function of $t$ as well as $x$, that is, using a normal mode form $w(x, z, t)=\hat{w}(z) e^{i k(x-c t)}+$ c.c. This would give the same steady-amplitude expressions we obtained but with $\bar{u}$ replaced by $\bar{u}-c$. We could then specify a spectrum of phase speeds $c$ and sum over $c$ to obtain a wave packet solution. A more realistic representation can be obtained by considering the gravity waves to be a perturbation with time-dependent amplitude. Sayed (2014) considered a single layer with stable stratification and gravity waves only and derived an analytical solution for the time-dependent gravity wave amplitude in the special case of the long-wave limit. From this, expressions for the momentum flux divergence and gravity wave drag can be obtained. Linear and nonlinear timedependent gravity wave solutions, for general aspect ratio, can be obtained numerically.

In the lower layer, however, the situation of steadyamplitude linear convective solutions and unstable stratification is physically unrealistic, and thus, it is necessary to retain the time-dependent and nonlinear terms. Sayed (2014) showed that in a layer with unstable stratification, the amplitude of the linear convective solution grows exponentially with time in the absence of viscosity and heat conduction. A nonlinear model is needed to allow the growing perturbation to modify and stabilize the background flow. This can be investigated numerically using the steady-amplitude solutions derived here as the initial state. 
Acknowledgments. This research was supported by the Natural Sciences and Engineering Research Council of Canada.

\section{APPENDIX}

\section{The Expressions for the Constants in (43)-(47)}

The constant $E$ in (43)-(47) is defined as $E=g F_{0} /\left(\rho_{0} \bar{u}^{2}\right)$. The other constants in (43)-(45) are

$$
\begin{aligned}
P= & -E e^{b h_{1}} e^{-b h} \frac{1}{b^{2}-\lambda^{2}}, \quad R=-\frac{2 b E}{b^{2}-\lambda^{2}}, \\
S= & E e^{-b h_{1}}\left(\frac{1}{b^{2}+m^{2}}-\frac{1}{b^{2}-\lambda^{2}}\right), \\
T= & E e^{-b h_{1}}\left(\frac{b}{b^{2}-\lambda^{2}}-\frac{b}{b^{2}+m^{2}}\right), \\
\alpha= & \frac{1}{\lambda \cosh \lambda h-i m \sinh \lambda h}[i m P \operatorname{sech} \lambda h \\
& \left.-\left(\frac{i m}{\lambda} \sinh \lambda h_{1}-\cosh \lambda h_{1}\right) R-i m S+T\right] .
\end{aligned}
$$

The other constants in (46) and (47) are

$$
\begin{aligned}
P_{0} & =-E e^{-b h} \frac{1}{b^{2}-\lambda^{2}}, \quad S_{0}=E\left(\frac{1}{b^{2}+m^{2}}-\frac{1}{b^{2}-\lambda^{2}}\right), \\
T_{0} & =-E\left(\frac{b}{b^{2}+m^{2}}+\frac{b}{b^{2}-\lambda^{2}}\right), \\
\alpha_{0} & =\frac{i m\left(P_{0} \operatorname{sech} \lambda h-S_{0}\right)+T_{0}}{\lambda \cosh \lambda h-i m \sinh \lambda h} .
\end{aligned}
$$

\section{REFERENCES}

Alexander, M. J., and L. Pfister, 1995: Gravity wave momentum flux in the lower stratosphere over convection. Geophys. Res. Lett., 22, 2029-2032, https://doi.org/10.1029/95GL01984.

— mean-flow forcing due to breaking gravity waves. J. Atmos. Sci., 56, 4167-4182, https://doi.org/10.1175/1520-0469(1999)056<4167: ASPOMF $>2.0 . \mathrm{CO} ; 2$.

_ J. R. Holton, and D. R. Durran, 1995: The gravity wave response above deep convection in a squall line simulation. J. Atmos. Sci., 52, 2212-2226, https://doi.org/10.1175/ 1520-0469(1995)052<2212:TGWRAD>2.0.CO;2.

_ J. H. Beres, and L. Pfister, 2000: Tropical stratospheric gravity wave activity and relationships to clouds. J. Geophys. Res., $\mathbf{1 0 5}$ 22 299-22 309, https://doi.org/10.1029/2000JD900326.

Alexander, S. P., T. Tsuda, Y. Kawatani, and M. Takahashi, 2008: Global distribution of atmospheric waves in the equatorial upper troposphere and lower stratosphere: COSMIC observations of wave mean flow interactions. J. Geophys. Res., 113, D24115, https://doi.org/10.1029/2008JD010039.

Baines, P., 1995: Topographic Effects in Stratified Flows. Cambridge University Press, $482 \mathrm{pp}$.
Batchelor, G. K., 1953: The condition for dynamical similarity of motions of a frictionless perfect-gas atmosphere. Quart. J. Roy. Meteor. Soc., 79, 224-235, https://doi.org/10.1002/ qj. 49707934004 .

Beres, J. H., 2004: Gravity wave generation by a three-dimensional thermal forcing. J. Atmos. Sci., 61, 1805-1815, https://doi.org/ 10.1175/1520-0469(2004)061<1805:GWGBAT>2.0.CO;2.

2005: Estimates of mesospheric gravity wave activity over convection from a global model. Adv. Space Res., 35, 19331939, https://doi.org/10.1016/j.asr.2005.04.087.

, M. J. Alexander, and J. R. Holton, 2002: Effects of tropospheric wind shear on the spectrum of convectively generated gravity waves. J. Atmos. Sci., 59, 1805-1824, https://doi.org/ 10.1175/1520-0469(2002)059<1805:EOTWSO >2.0.CO;2.

,$- \ldots$, and,- 2004 : A method of specifying the gravity wave spectrum above convection based on latent heating properties and background wind. J. Atmos. Sci., 61, 324-337, https://doi.org/ 10.1175/1520-0469(2004)061<0324:AMOSTG>2.0.CO;2.

- R. R. Garcia, R. B. Boville, and F. Sassi, 2005: Implementation of a gravity wave source spectrum parameterization dependent on the properties of convection in the Whole Atmosphere Community Climate Model (WACCM). J. Geophys. Res., 110, D10108, https://doi.org/10.1029/2004JD005504.

Bergman, J. W., and M. L. Salby, 1994: Equatorial wave activity derived from fluctuations in observed convection. J. Atmos. Sci., 51,3791-3806, https://doi.org/10.1175/1520-0469(1994)051<3791: EWADFF $>2.0 . \mathrm{CO} ; 2$.

Booker, J. R., and F. P. Bretherton, 1967: The critical layer for gravity waves in a shear flow. J. Fluid Mech., 27, 513-539, https://doi.org/10.1017/S0022112067000515.

Chun, H.-Y., and J.-J. Baik, 1998: Momentum flux by thermally induced internal gravity waves and its approximation for largescale models. J. Atmos. Sci., 55, 3299-3310, https://doi.org/ 10.1175/1520-0469(1998)055<3299:MFBTII > 2.0.CO;2.

, and - 2002: An updated parameterization of convectively forced gravity wave drag for use in large-scale models. J. Atmos. Sci., 59, 1006-1017, https://doi.org/10.1175/ 1520-0469(2002)059<1006:AUPOCF>2.0.CO;2.

—, I.-S. Song, J.-J. Baik, and Y.-J. Kim, 2004: Impact of a convectively forced gravity wave drag parameterization in NCAR CCM3. J. Climate, 17, 3530-3547, https://doi.org/ 10.1175/1520-0442(2004)017<3530:IOACFG>2.0.CO;2.

Clark, T. L., T. Hauf, and J. P. Kuettner, 1986: Convectively forced internal gravity waves: Results from two-dimensional numerical experiments. Quart. J. Roy. Meteor. Soc., 112, 899925, https://doi.org/10.1002/qj.49711247402.

Dutta, G., M. C. Ajay Kumar, P. Vinay Kumar, M. Venkat Ratnam, M. Chandrashekar, Y. Shibagaki, M. Salauddin, and H. A. Basha, 2009: Characteristics of high-frequency gravity waves generated by tropical deep convection: Case studies. J. Geophys. Res., 114, D18109, https://doi.org/10.1029/2008JD011332.

Fovell, R., D. Durran, and J. R. Holton, 1992: Numerical simulations of convectively generated stratospheric gravity waves. J. Atmos. Sci., 49, 1427-1442, https://doi.org/10.1175/ 1520-0469(1992)049<1427:NSOCGS > 2.0.CO;2.

Fritts, D. C., and M. J. Alexander, 2003: Gravity wave dynamics and effects in the middle atmosphere. Rev. Geophys., 41, 1003, https://doi.org/10.1029/2001RG000106.

Gill, A. E., 1982: Atmosphere-Ocean Dynamics. Academic Press, $662 \mathrm{pp}$.

Goldstein, S., 1931: On the stability of superposed streams of fluids of different densities. Proc. Roy. Soc. London, 132A, 524-548, https://doi.org/10.1098/rspa.1931.0116. 
Hayashi, Y., 1976: Non-singular resonance of equatorial waves under the radiation condition. J. Atmos. Sci., 33, 183-201, https://doi.org/10.1175/1520-0469(1976)033<0183: NSROEW $>2.0 . \mathrm{CO} ; 2$.

Hines, C. O., 1997: Doppler-spread parameterization of gravitywave momentum deposition in the middle atmosphere. Part 2: Broad and quasi monochromatic spectra, and implementation. J. Atmos. Sol.-Terr. Phys., 59, 387-400, https://doi.org/10.1016/ S1364-6826(96)00080-6.

Holton, J. R., 2004: An Introduction to Dynamic Meteorology. 4th ed. Elsevier, 535 pp.

— J. Beres, and X. Zhou, 2002: On the vertical scale of gravity waves excited by localized thermal forcing. J. Atmos. Sci., $\mathbf{5 9}$, 2019-2023, https://doi.org/10.1175/1520-0469(2002)059<2019: OTVSOG $>2.0 . \mathrm{CO} ; 2$.

Jewtoukoff, V., R. Plougonven, and A. Hertzog, 2013: Gravity waves generated by deep tropical convection: Estimates from balloon observations and mesoscale simulations. J. Geophys. Res. Atmos., 118, 9690-9707, https://doi.org/10.1002/jgrd.50781.

Kovalam, S., R. A. Vincent, and P. Love, 2006: Gravity waves in the equatorial MLT region. J. Atmos. Sol.-Terr. Phys., 68, 266-282, https://doi.org/10.1016/j.jastp.2005.05.009.

Kuester, M. A., M. J. Alexander, and E. A. Ray, 2008: A model study of gravity waves over Hurricane Humberto (2001). J. Atmos. Sci., 65, 3231-3246, https://doi.org/10.1175/2008JAS2372.1.

Lane, T. P., and M. W. Moncrieff, 2008: Stratospheric gravity waves generated by multiscale tropical convection. J. Atmos. Sci., 65, 2598-2614, https://doi.org/10.1175/2007JAS2601.1.

_ - M. J. Reeder, and T. L. Clark, 2001: Numerical modeling of gravity wave generation by deep tropical convection. J. Atmos. Sci., 58, 1249-1274, https://doi.org/10.1175/1520-0469(2001)058<1249: NMOGWG $>2.0 . \mathrm{CO} ; 2$.

Lindzen, R. S., 1981: Turbulence and stress owing to gravity wave and tidal breakdown. J. Geophys. Res., 86, 9707-9714, https:// doi.org/10.1029/JC086iC10p09707.

McLandress, C. D., M. J. Alexander, and L. Wu, 2000: Microwave Limb Sounder observations of gravity waves in the stratosphere: A climatology and interpretation. J. Geophys. Res., 105, 11 947-11 967, https://doi.org/10.1029/ 2000JD900097.

Ogura, Y., and N. A. Phillips, 1962: Scale analysis of deep and shallow convection in the atmosphere. J. Atmos. Sci., 19, 173-179, https://doi.org/10.1175/1520-0469(1962)019<0173: SAODAS $>2.0 . \mathrm{CO} ; 2$.

Pandya, R. E., and M. J. Alexander, 1999: Linear stratospheric gravity waves above convective thermal forcing. J. Atmos. Sci., 56, 2434-2446, https://doi.org/10.1175/1520-0469(1999)056<2434: LSGWAC $>2.0 . \mathrm{CO} ; 2$.

Pfister, L., W. Starr, R. Craig, and M. Loewenstein, 1986: Smallscale motions observed by aircraft in the tropical lower stratosphere: Evidence for mixing and its relationship to largescale flows. J. Atmos. Sci., 43, 3210-3225, https://doi.org/ 10.1175/1520-0469(1986)043<3210:SSMOBA > 2.0.CO;2.
— S. Scott, M. Loewenstein, S. Bowen, and M. Legg, 1993: Mesoscale disturbances in the tropical stratosphere excited by convection: Observations and effects on the stratospheric momentum budget. J. Atmos. Sci., 50, 1058-1075, https://doi.org/ 10.1175/1520-0469(1993)050<1058:MDITTS > 2.0.CO;2.

Piani, C., D. Durran, M. J. Alexander, and J. R. Holton, 2000: A numerical study of three-dimensional gravity waves triggered by deep tropical convection and their role in the dynamics of the QBO. J. Atmos. Sci., 57, 3689-3702, https://doi.org/ 10.1175/1520-0469(2000)057<3689:ANSOTD>2.0.CO;2.

Salby, M. L., and R. R. Garcia, 1987: Transient response to localized episodic heating in the tropics. Part I: Excitation and short-time near-field behavior. J. Atmos. Sci., 44, 458-498, https://doi.org/ 10.1175/1520-0469(1987)044<0458:TRTLEH >2.0.CO;2.

Sang, J., 1991: On formation of convective roll vortices by internal gravity waves: A theoretical study. Meteor. Atmos. Phys., 46, 15-28, https://doi.org/10.1007/BF01026620.

Sato, K., 1992: Vertical wind disturbances in the afternoon of midsummer revealed by the MU radar. Geophys. Res. Lett., 19 , 1943-1946, https://doi.org/10.1029/92GL02244.

Sayed, A. A. M., 2014: Internal gravity waves and convection generated by a thermal forcing in the atmosphere. Ph.D. thesis, Carleton University, 199 pp.

Song, I. S., H. Y. Chun, and T. P. Lane, 2003: Generation mechanisms of convectively forced internal gravity waves and their propagation to the stratosphere. J. Atmos. Sci., 60, 1960-1980, https://doi.org/ 10.1175/1520-0469(2003)060<1960:GMOCFI >2.0.CO;2.

Sutherland, B. R., 2010: Internal Gravity Waves. Cambridge University Press, 377 pp.

Taylor, G. I., 1931: Effect of variation in density on the stability of superposed streams of fluid. Proc. Roy. Soc. London, 132A, 499-523, https://doi.org/10.1098/rspa.1931.0115.

Taylor, M. J., and Coauthors, 2009: Characteristics of mesospheric gravity waves near the magnetic equator, Brazil, during the SpreadFEx campaign. Ann. Geophys., 27, 461-472, https://doi.org/10.5194/angeo-27-461-2009.

Tsuda, T., S. Kato, T. Yokoi, T. Inoue, M. Yamamoto, T. VanZandt, S. Fukao, and T. Sato, 1990: Gravity waves in the mesosphere observed with the middle and upper atmosphere radar. Radio Sci., 25, 1005-1018, https://doi.org/ 10.1029/RS025i005p01005.

Venkateswara Rao, N., Y. Shibagaki, and T. Tsuda, 2011: Diurnal variation of short-period $(20-120 \mathrm{~min})$ gravity waves in the equatorial mesosphere and lower thermosphere and its relation to deep tropical convection. Ann. Geophys., 29, 623-629, https://doi.org/10.5194/angeo-29-623-2011.

Vincent, R. A., and M. J. Alexander, 2000: Gravity waves in the tropical lower stratosphere: An observational study of seasonal and interannual variability. J. Geophys. Res., 105, 17 971-17 982, https://doi.org/10.1029/2000JD900196.

Wurtele, M. G., R. D. Sharman, and A. Datta, 1996: Atmospheric lee waves. Annu. Rev. Fluid Mech., 28, 429-476, https://doi.org/ 10.1146/annurev.fl.28.010196.002241. 